\title{
Roles of vertical distributions of atmospheric transient eddy dynamical forcing and diabatic heating in midlatitude unstable air-sea interaction
}

Jiabei Fang

Nanjing University

Lilan Chen

Nanjing University

Xiu-Qun Yang ( $\nabla$ xqyang@nju.edu.cn )

Nanjing University https://orcid.org/0000-0003-3716-9152

\section{Original Article}

Keywords: Midlatitude air-sea interaction, diabatic heating, transient eddy vorticity forcing, baroclinic atmosphere model

Posted Date: February 9th, 2021

DOI: https://doi.org/10.21203/rs.3.rs-198305/v1

License: (c) (i) This work is licensed under a Creative Commons Attribution 4.0 International License. Read Full License

Version of Record: A version of this preprint was published at Climate Dynamics on August 9th, 2021. See the published version at https://doi.org/10.1007/s00382-021-05912-8. 
1 Roles of vertical distributions of atmospheric transient eddy

2 dynamical forcing and diabatic heating in midlatitude unstable

3 air-sea interaction

4

Jiabei Fang, Lilan Chen, Xiu-Qun Yang

6

CMA-NJU Joint Laboratory for Climate Prediction Studies, School of Atmospheric

Sciences, Nanjing University, Nanjing, China

8

9

Correspondence to:

11

Dr. Xiu-Qun Yang, xqyang@nju.edu.cn,

12

Dr. Jiabei Fang, fangjb@nju.edu.cn

13

14 
Abstract

Atmospheric transient eddy dynamical forcing (TEDF)-driven midlatitude unstable air-sea interaction has recently been recognized as a crucial positive feedback for the maintenance of the extratropical decadal variabilities. Our previous theoretical work by Chen et al. (2020) characterizes such an interaction with building an analytical midlatitude barotropic atmospheric model coupled to a simplified upper oceanic model. This study firstly extends the analytical model to a two-layer quasi-geostrophic baroclinic atmospheric model coupled to a simplified upper oceanic model and then identifies the roles of vertical distributions of atmospheric TEDF and diabatic heating in midlatitude unstable air-sea interaction. It is found that the midlatitude air-sea coupling through atmospheric TEDF and diabatic heating with more realistic vertical profile destabilizes the oceanic Rossby wave mode over the entire range of zonal wavelengths, and the most unstable mode exhibits an equivalent barotropic structure with geopotential lows (highs) over cold (warm) water. The spatial configuration structure and period of the most unstable coupled mode are more consistent with the observation than those from the previous model. Although either TEDF or diabatic heating alone can lead to unstable air-sea interaction, the former is dominant to the instability. TEDF in both higher and lower layers can cause unstable coupled mode individually, while the lower-layer forcing stimulates instability more effectively. Surface diabatic heating always destabilizes the coupled mode, while the mid-level heating always decays the coupled mode. Moreover, the influences of oceanic adjustment processes, air-sea coupling strength and background zonal wind on the unstable coupled mode are also discussed. The results of this study further prove the TEDF-driven positive feedback mechanism in midlatitude air-sea interaction proposed by recent observational and numerical experiment studies.

Keywords: Midlatitude air-sea interaction, diabatic heating, transient eddy vorticity forcing, baroclinic atmosphere model 


\section{Introduction}

Observational studies have revealed that there is a significant decadal variability in the midlatitude North Pacific ocean-atmosphere system (Trenberth 1990; Graham et al. 1994; Minobe 1997; Mantua et al. 1997; Enfield and Mestas-Nunez 1999; Zhu and Yang 2003; Fang et al. 2006). The decadal mode of North Pacific SST anomalies, also known as the Pacific Decadal Oscillation (PDO), is well correlated with the atmospheric circulation anomalies that exhibit an equivalent barotropic structure in the vertical direction, with geopotential lows (highs) above cold (warm) water (Kushnir et al. 2002; Namias and Cayan 1981; Fang and Yang 2016). Such an equivalent barotropic cold/ trough (warm/ridge) structure is also the typical feature of midlatitude climate variabilities on decadal time scale (Cayan 1992; Deser and Blackman 1993).

As we know, to generate an oscillation, a positive feedback mechanism is needed to make the initial perturbation develop, and a delayed negative feedback mechanism is also needed to make the anomalous phase transition. For PDO, the negative feedback mechanism is thought to be the slow upper-ocean adjustment processes involving the oceanic gyre (Latif and Barnett 1994; Fang et al. 2006), the subduction (Gu and Philander 1997), and the oceanic Rossby wave propagation (Qiu et al. 2007). The decadal time scale of PDO is determined by the adjustment period. While the positive feedback mechanism is considered to be the local large-scale ocean-atmosphere interaction in the middle latitudes (Latif and Barnett 1994, 1996; Latif 1999; Miller and Schneider 2000; Robertson 1996; Zorita and Frankignoul 1997). Though a number of observations, theoretical analyses and GCM simulations have provided evidence for the active feedback of extratropical SST on large-scale atmospheric circulation (Saravanan and McWilliams 1997, 1998; Neelin and Weng 1999; Latif and Barnett 1996; Liu and Wu 2004; Zhong et al. 2008; Zhu et al. 2008; Fang and Yang 2011), the mechanism responsible for the midlatitude air-sea interaction remains unclear for a long time. It is 
primarily because the processes by which the midlatitude sea surface temperature (SST) anomaly affects the seasonal-mean atmospheric anomaly has not been fully understood.

Different from the tropical atmospheric circulation that is mainly driven by the SST-induced convective diabatic heating, the midlatitude atmospheric circulation is both thermally- and eddy-driven. Since the atmospheric stratification in the middle latitudes is stable, the SST-induced diabatic heating, mostly through sensible heat transportation and latent heat release, is relatively weak and mainly confined to the lower troposphere. On the other hand, the midlatitude atmosphere is strongly baroclinic, and the synoptic transient eddies are the most active over midlatitude oceans, particularly over the midlatitude oceanic frontal zones, forming stormtracks (Ren et al. 2010; Chu et al. 2013; Liu et al. 2014; Nakamura et al. 2004; Small et al. 2008; Wang et al. 2017). The nonlinear interaction of transient eddies can systematically transport heat and momentum, driving and maintaining atmospheric circulations in the middle latitudes (Ren et al. 2011; Xiang and Yang 2012; Zhang et al. 2012; Nie et al. 2013, 2014). From the perspective of quasi-geostrophic potential vorticity (QGPV) dynamics, the seasonal-mean PV sources include the diabatic heating, the transient eddy heating, and the transient eddy vorticity forcing (Lau and Holopainen 1984; Fang and Yang 2016). The latter two items that are caused by the convergence of the heat and vorticity transport by transient eddies, respectively, can be considered as indirect forcing sources of atmospheric circulation. Therefore, the midlatitude SST anomalies can affect the atmosphere by changing both the diabatic heating and the transient eddy forcing.

Fang and Yang (2016) identified the distribution features of the PDO-related atmospheric forcing terms and quantitatively diagnosed their effect on the atmosphere. Based on these analyses, a hypothesis on the positive feedback mechanism for midlatitude unstable air-sea interaction in the North Pacific was further proposed. An initial midlatitude surface westerly anomaly accompanied with intensified Aleutian low tends to force a negative SST anomaly by increasing upward surface heat fluxes and 
driving southward Ekman current anomaly. Then, the basin-scale SST cooling tends to increase the meridional SST gradient in its southern flank, thus enhancing the subtropical oceanic front. As an adjustment to the enhanced oceanic front, the low-level atmospheric baroclinicity tends to be strengthened, and more transient eddies are generated, resulting in more meridional thermal and momentum transportation. Although all the diabatic heating, transient eddy heating and transient eddy vorticity forcing are increased during these processes, only the atmospheric response to the transient eddy vorticity forcing tends to be barotropic. The vorticity forcing that dominates the total atmospheric forcing tends to produce an equivalent barotropic atmospheric low pressure that intensifies the initial anomalies of the midlatitude surface westerly and Aleutian low. Therefore, the midlatitude ocean-atmosphere interaction, in which the oceanic front and the atmospheric transient eddy are the indispensable ingredients, can provide a positive feedback mechanism for the development and maintenance of the observed decadal anomalies in the midlatitude North Pacific oceanatmosphere system. This hypothesis has also been confirmed by the later observational and numerical simulation studies (Wang et al., 2017; Wang et al. 2019; Tao et al. 2020; Zhang et al. 2020).

Since the transient eddy dynamical feedback is an important process for the ocean to influence the atmosphere, in our recent work (Chen et al, 2020, hereinafter CYF2020), an analytical coupled air-sea model including atmospheric vorticity forcing is established to investigate its contribution to midlatitude unstable air-sea interaction. The transient eddy vorticity forcing is parameterized to be linearly proportional to the meridional second-order derivative of SST on the base of observational analyses. Considering the prominent characteristics of the equivalent barotropic structure of the midlatitude atmospheric circulation, for simplicity, the atmospheric model is assumed to be barotropic. It is found that the oceanic Rossby wave stimulated by air-sea coupling can be unstable within a wide range of wavelengths. The configuration structure of the 
most unstable mode is similar to that of the observed decadal variability. Although both surface diabatic heating and transient eddy vorticity forcing can cause unstable mode, the latter plays a dominant role. This results also provide theoretical support for the midlatitude ocean-atmosphere interaction hypothesis.

However, in the previous theoretical research, the assumption of barotropic atmosphere is simple. Actually, both diabatic heating and transient eddy vorticity forcing have vertical variations. Some studies using linear baroclinic model show that the atmospheric response is sensitive to the vertical structure of the idealized heat source. To low-level heating, the atmosphere tends to produce a barotropic lowpressure response, while to the mid-level heat source, the atmospheric response tends to be baroclinic (Qiu et al. 2014; Hoskins and Karoly 1981; Fang and Yang 2011). Moreover, the influence of vertical distribution of the transient eddy vorticity forcing on the atmospheric response is still unclear.

Therefore, in the present study, the atmospheric component of the analytical midlatitude coupled air-sea model in CYF2020 is extended to a two-layer baroclinic quasi-geostrophic model, and the oceanic component remains a one and a half-layer upper ocean model. The vertical structures of diabatic heating and transient eddy vorticity forcing are both taken into account to further explore their relative contributions to midlatitude unstable ocean-atmosphere interaction. The paper is organized as follows. Description of the analytical coupled model are presented in Section 2. The dynamical features of midlatitude air-sea coupled mode and the relative contributions of diabatic heating and transient eddy vorticity forcing with difference vertical profiles are examined in Section 3 and Section 4, respectively. Comparison with the results of barotropic model is presented in Section 5. Influence of other processes and factors on unstable coupled mode such as the oceanic adjustment processes, the air-sea coupling intensity and the background atmospheric flow are discussed in Section 6. The final section is devoted to the conclusions and discussion. 


\section{An extended analytical midlatitude coupled air-sea model}

150 Figure 1 shows the diagram of the extended analytical coupled model used in this study.

151 The atmospheric component is described by a two-layer baroclinic quasi-geostrophic model with a shallow boundary layer. The oceanic component remains a quasi-

153 geostrophic upper-ocean model, as in CYF2020, which consists of a constant-depth 154 mixed layer, a thin entrainment layer and a thermocline layer. Both the atmosphere and ocean are governed by their respective quasi-geostrophic potential vorticity (QGPV) equations on a midlatitude beta plane.

For the atmosphere, the diabatic heating is imagined to occur both at the top of boundary layer (surface heating, $Q_{s}$ ) and between the two layers (mid-level heating, $Q)$. The transient eddy vorticity forcing $\left(F_{\text {eddy }}\right)$ exists in both layers. Since the adjustment time scale of the atmosphere is much shorter than that of the ocean, the atmospheric fluctuations are assumed to be a steady-state response to the forcing sources. Therefore, the seasonal mean QGPV equations for the two atmospheric layers can be written as

$$
\begin{gathered}
J\left(\bar{\psi}_{1}, \bar{q}_{1}\right)+r \nabla^{2} \bar{\psi}_{1}=\bar{F}_{e d d y 1}-\frac{g H_{a} \bar{Q}}{2 f L_{a}^{2}} \quad \text { and } \\
J\left(\bar{\psi}_{2}, \bar{q}_{2}\right)+r \nabla^{2} \bar{\psi}_{2}=\bar{F}_{e d d y 2}+\frac{g H_{a}\left(\bar{Q}-\bar{Q}_{s}\right)}{2 f L_{a}^{2}},
\end{gathered}
$$

167 where the overbar denotes seasonal mean, $q$ is the potential vorticity defined by

$$
\bar{q}_{1}=\nabla^{2} \bar{\psi}_{1}+\beta y-
$$

$$
\bar{q}_{2}=\nabla^{2} \bar{\psi}_{2}+\beta y+\frac{1}{L_{a}^{2}}\left(\bar{\psi}_{1}-\bar{\psi}_{2}\right)
$$

$172 \psi$ is the stream function, $J(a, b)=\frac{\partial a}{\partial x} \frac{\partial b}{\partial y}-\frac{\partial a}{\partial y} \frac{\partial b}{\partial x}$ is the Jacobi operator, $L_{a}=$

$173\left(N_{a} H_{a}\right) /(2 f)$ is the atmospheric baroclinic Rossby radius of deformation with $N_{a}$ 
the atmospheric Brunt-Väisälä buoyancy frequency, and $H_{a}$ is the depth of the free troposphere. According to the equations, the seasonal mean atmospheric circulation is driven by both diabatic heat forcing (the second term on the right hand) and transient eddy vorticity forcing $\left(\bar{F}_{\text {eddy }}\right)$. The former term is proportional to the vertical gradient of diabatic heating, while $\bar{F}_{e d d y}=-\nabla \cdot \overline{\vec{V}}_{h}^{\prime} \zeta^{\prime}$ represents the seasonal mean vorticity transportation by synoptic transient eddies (denoted by primes). $r \nabla^{2} \bar{\psi}$ indicates the damping effect that is proportional to the relative vorticity, and $r$ is the diffusive coefficient with the characteristic time scale $r^{-1}=5$ days (Pedlosky 1970).

If we simply suppose that the basic state is horizontally uniform zonal flow with vertical shear, the seasonal mean atmospheric streamfunction can be expressed as the sum of a basic flow (climatological mean) and a perturbation (seasonal anomaly) like $\bar{\psi}_{1}=\psi_{1}-U_{1} y, \bar{\psi}_{2}=\psi_{2}-U_{2} y$. Further decomposing the atmospheric circulation into barotropic and baroclinic components by defining

$$
\begin{aligned}
& \widehat{\psi}=\psi_{1}+\psi_{2} ; \quad \tilde{\psi}=\psi_{1}-\psi_{2} \\
& \widehat{U}=U_{1}+U_{2} ; \quad \widetilde{U}=U_{1}-U_{2},
\end{aligned}
$$

we can derive the linearized barotropic and baroclinic PV perturbation equations as

$$
\widehat{U} \frac{\partial}{\partial x} \nabla^{2} \hat{\psi}+\hat{\beta} \frac{\partial \widehat{\psi}}{\partial x}+\widetilde{U} \frac{\partial}{\partial x} \nabla^{2} \tilde{\psi}+2 r \nabla^{2} \hat{\psi}=2 F_{e d d y 1}+2 F_{e d d y 2}-\frac{g H_{a} Q_{s}}{f L_{a}^{2}}
$$

$$
\begin{aligned}
\widehat{U} \frac{\partial}{\partial x}\left\{\nabla^{2} \tilde{\psi}-\frac{2}{L_{a}^{2}} \tilde{\psi}\right\}+\tilde{\beta} \frac{\partial \widehat{\psi}}{\partial x}+\widetilde{U} \frac{\partial}{\partial x} \nabla^{2} \widehat{\psi}+\hat{\beta} \frac{\partial \widetilde{\psi}}{\partial x}+2 r \nabla^{2} \tilde{\psi}= \\
2 F_{e d d y 1}-2 F_{e d d y 2}-\frac{2 g H_{a} Q}{f L_{a}^{2}}+\frac{g H_{a} Q_{s}}{f L_{a}^{2}}
\end{aligned}
$$

where $\hat{\beta}=2 \beta, \tilde{\beta}=2 \widetilde{U} / L_{a}^{2}$. It should be noted that the surface diabatic heating and the atmospheric transient eddy vorticity forcing in each layer drive the barotropic and baroclinic components together, whereas the mid-level heating only acts on the baroclinic component. 
As documented in CYF2020, the anomalous transient eddy vorticity forcing is proportional to the meridional second order derivative of SST anomaly. Thus here the vorticity forcing in the upper layer is specified equal to that in the barotropic coupled model (CYF2020) given by $F_{e d d y 1}=\gamma \partial^{2} T_{1} / \partial y^{2}$, with $T_{1}$ the sea surface temperature anomaly. Meanwhile, following Fang and Yang (2011) as well as Shutts (1987), the mid-level heating is prescribed to have the form of Newtonian relaxation of the atmospheric potential temperature perturbation at middle level $\theta_{a}$ to a radiativeconvective equilibrium temperature anomaly $\theta_{a}^{*}$, given by

$$
Q=-\gamma_{a}\left(\frac{\theta_{a}}{\theta_{a o}}-\frac{\theta_{a}^{*}}{\theta_{a o}}\right)=-\gamma_{a}\left(\frac{2 f}{g H_{a}} \tilde{\psi}-\frac{\theta_{a}^{*}}{\theta_{a o}}\right)
$$

207 Here $\theta_{a}$ has been converted to $\tilde{\psi}$ using the thermal wind relation, $\theta_{a o}$ is a typical 208 value of $\theta_{a}, \gamma_{a}$ is the atmospheric air-sea heat exchange parameter, and $\theta_{a}^{*}$ is set to 209 be the function of SST anomaly. For simplicity, it is assumed that $\theta_{a}^{*}=T_{1}$.

210 Based on the observational analysis on the vertical distribution of the anomalous 211 diabatic heating and transient eddy vorticity forcing in North Pacific region (Fig. 2b and c, quoted from CYF2020), the surface heating is estimated to be twice as large as 213 that of mid-level heating $\left(Q_{s}=\gamma_{2} Q, \gamma_{2}=2\right)$, and the transient eddy vorticity forcing 214 in the lower atmosphere is estimated to be 0.2 times of that in the upper atmosphere $215\left(F_{\text {eddy } 2}=\delta_{2} F_{\text {eddy1 } 1}, \delta_{2}=0.2\right)$. Hence the atmospheric equations (7)-(8) can be 216 rewritten as

$$
\begin{array}{r}
\widehat{U} \frac{\partial}{\partial x} \nabla^{2} \hat{\psi}+\hat{\beta} \frac{\partial \widehat{\psi}}{\partial x}+\widetilde{U} \frac{\partial}{\partial x} \nabla^{2} \tilde{\psi}+2 r \nabla^{2} \hat{\psi} \\
=2 \gamma\left(1+\delta_{2}\right) \frac{\partial^{2}}{\partial y^{2}} T_{1}+\frac{2 \gamma_{a} \gamma_{2}}{L_{a}^{2}}\left(\tilde{\psi}-\frac{1}{r_{a}} T_{1}\right) \\
\widehat{U} \frac{\partial}{\partial x}\left\{\nabla^{2} \tilde{\psi}-\frac{2}{L_{a}^{2}} \tilde{\psi}\right\}+\tilde{\beta} \frac{\partial \widehat{\psi}}{\partial x}+\widetilde{U} \frac{\partial}{\partial x} \nabla^{2} \hat{\psi}+\hat{\beta} \frac{\partial \widetilde{\psi}}{\partial x}+2 r \nabla^{2} \tilde{\psi} \\
=2 \gamma\left(1-\delta_{2}\right) \frac{\partial^{2} T_{1}}{\partial y^{2}}+\frac{2 \gamma_{a}\left(2-\gamma_{2}\right)}{L_{a}^{2}}\left(\tilde{\psi}-\frac{1}{r_{a}} T_{1}\right),
\end{array}
$$

Where $r_{a} \equiv\left(2 f \theta_{a o}\right) /\left(g H_{a}\right)$. 

and in Fang and Yang (2011). The upper-layer motion represented by the first

224 baroclinic Rossby mode is controlled by a linear QGPV equation with a rest basic state 225 driven by the curl of the surface wind stress. The evolution of SST is determined by the 226 physical processes including horizontal advection, vertical entrainment and heat 227 exchange at air-sea interface. The governing equations are given by

$$
-\frac{1}{L_{o}^{2}} \frac{\partial \psi_{o}}{\partial t}+\beta \frac{\partial \psi_{o}}{\partial x}=\alpha \nabla^{2}(\hat{\psi} / 2-\tilde{\psi})
$$

$$
\frac{\partial T_{1}}{\partial t}=c_{1} \psi_{o}-c_{2} T_{1}-c_{3} W_{e}+c_{4} \frac{\partial \psi_{o}}{\partial x}+c_{4} \frac{H-H_{1}}{H} v_{s}-\gamma_{o}\left(T_{1}-r_{a} \tilde{\psi}\right)
$$

231 where $\psi_{o}$ is the upper-layer oceanic geostrophic streamfunction, $L_{o}$ is the oceanic

232 baroclinic Rossby radius of deformation, $c_{1}, c_{2}, c_{3}$ and $c_{4}$ are all positive coefficients 233 relevant to advection and entrainment processes, and $\gamma_{o}$ is the oceanic air-sea heat 234 exchange parameter.

Therefore, equations (9)-(12) constitute the final closed equations for the coupled ocean-atmosphere model. Compared with the coupled model used in Fang and Yang (2011), the mechanism of transient eddy dynamical feedback is introduced in the present model. Also, the coupled model with barotropic atmosphere in CYF2020 can be regarded as a special case of the present model.

\section{Features and dynamics of the unstable midlatitude coupled modes}

241 The perturbations of the atmospheric and oceanic variables are assumed to be limited 242 within a $[0, \pi]$ channel in $Y$ direction, and to satisfy the standard plane wave solution 243 in $X$ direction with the form

$$
\left(\hat{\psi}, \tilde{\psi}, \psi_{o}, T_{1}\right)=\left(\hat{\psi}^{\prime \prime}, \tilde{\psi}^{\prime \prime}, \psi_{o}^{\prime \prime}, T_{1}^{\prime \prime}\right) e^{i(k x-\sigma t)} \text { sinly }
$$

245 Here $\hat{\psi}^{\prime \prime}, \tilde{\psi}^{\prime \prime}, \psi_{o}^{\prime \prime}$ and $T_{1}^{\prime \prime}$ are the amplitudes of these variables, $\sigma$ is the complex 246 frequency, $k$ and $l$ are the horizontal wavenumbers in $x$ and $y$ directions, 
respectively. Inserting (13) into equations (9)-(12), we can obtain the following dispersion relations of the coupled modes:

$$
\begin{gathered}
\sigma_{ \pm}=\frac{1}{2}\left\{\omega_{r}-i c_{2}-i \gamma_{o}+\frac{\left[2 \gamma l^{2}\left(1+\delta_{2}\right)+\frac{2 \gamma_{a} \gamma_{2}}{L_{a}^{2}} r_{a}\right]\left[\gamma_{o} r_{a}-\left[c_{3}^{\prime} f K^{2}+c_{4}^{\prime}\left(r_{s} k+f l\right) i\right] \frac{\alpha\left(\frac{\mu}{2}+1\right)}{\left(f^{2}+r_{s}^{2}\right)}\right]}{\mu k\left(-\widehat{U} K^{2}+\widehat{\beta}-\frac{2 r K^{2}}{i k}\right)+\widetilde{U} k K^{2}+\frac{2 \gamma a \gamma_{2}}{L_{a}^{2} i}}\right\} \\
\pm i\left\{-\frac{1}{4}\left(\omega_{r}+i c_{2}+i \gamma_{o}-\frac{\left[2 \gamma l^{2}\left(1+\delta_{2}\right)+\frac{2 \gamma_{a} \gamma_{2}}{L_{a}^{2}} r_{a}\right]\left[\gamma_{o} r_{a}-\left[c_{3}^{\prime} f K^{2}+c_{4}^{\prime}\left(r_{s} k+f l\right) i\right] \frac{\alpha\left(\frac{\mu}{2}+1\right)}{\left(f^{2}+r_{S}^{2}\right)}\right]}{\mu k\left(-\widehat{U} K^{2}+\widehat{\beta}-\frac{2 r K^{2}}{i k}\right)+\widetilde{U} k K^{2}+\frac{2 \gamma_{a} \gamma_{2}}{L_{a}^{2} i}}\right)^{2}-\left(c_{1}+\right.\right.
\end{gathered}
$$

$\left.C_{4} i k\right) \frac{\alpha K^{2} L_{o}^{2}\left(\frac{\mu}{2}+1\right)\left[2 \gamma l^{2}\left(1+\delta_{2}\right)+\frac{2 \gamma_{a} \gamma_{2}}{L_{a}^{2}} r_{a}\right]}{\left[\mu i k\left(-\widehat{U} K^{2}+\widehat{\beta}-\frac{2 \gamma K^{2}}{i k}\right)+\widetilde{U} i k K^{2}+\frac{2 \gamma_{a} \gamma_{2}}{L_{a}^{2}}\right\}^{\frac{1}{2}}}$,

252 where $\omega_{r} \equiv-k \beta L_{\mathrm{o}}^{2}$ is the frequency of free oceanic baroclinic Rossby wave, $K^{2}=$ $253 k^{2}+l^{2}$ represents the square of total wavenumber, $c_{3}^{\prime}=c_{3}\left(H-H_{1}\right)$ and $c_{4}^{\prime}=$ $c_{4}\left(H-H_{1}\right) / H_{1}$ are both positive coefficients, and

$$
\mu=\frac{\widetilde{U} K^{2}+\widehat{U} K_{a}^{2}-\widehat{\beta}+\frac{2 r K^{2}}{i k}+\frac{2 \gamma l^{2} L_{a}^{2} r_{a}}{\gamma_{a} \gamma_{2}}\left(-\widehat{U} K_{a}^{2}+\widehat{\beta}-\frac{2 r K^{2}}{i k}+\frac{2 \gamma_{a} \gamma_{2}}{L_{a}^{2} i k}\right)}{\widehat{U} K^{2}-\widehat{\beta}+\frac{2 r K^{2}}{i k}+\widetilde{U} K^{2}-\widetilde{\beta}+\frac{2 \gamma l^{2} L_{a}^{2} r_{a}}{\gamma_{a} \gamma_{2}}\left(-\widetilde{U} K^{2}+\widetilde{\beta}\right)}
$$

measures the relative strength of barotropic and baroclinic modes as $\quad \hat{\psi}=-\mu \tilde{\psi}$.

The two solutions of (14) corresponds to two coupled modes caused by midaltitude air-sea interaction. The real part of $\sigma$ represents the frequency of the coupled mode and the imaginary part indicates the growth rate of the amplitude of coupled mode. Therefore, the coupled mode will be unstable if the imaginary part of $\sigma$ is positive. Given the same parameter values as in CYF2020 (see Table 1), except that the basic zonal wind for upper and lower atmosphere are $U_{1}=17 \mathrm{~ms}^{-1}$ and $U_{2}=8 \mathrm{~ms}^{-1}$ respectively. The frequency and growth rate of the two coupled modes can be calculated as a function of zonal wavelength. The frequency curve of the first mode (the blue line in Fig.3a) is very close to that of the free oceanic Rossby wave (the red line), thus the

267 first coupled mode $\left(\sigma_{+}\right)$is essentially a coupled oceanic Rossby wave mode modified 268 by air-sea interaction. The coupled oceanic Rossby wave propagates westward and 269 destabilizes over the whole range of wavelengths (Fig. 3b). The other coupled mode 
$270\left(\sigma_{-}\right)$corresponds to a eastward-propagating decay mode since its growth rate is always 271 negative within entire zonal wavelengths (Figure not shown). Because our focus in this 272 study is mainly on the unstable air-sea interaction, the damping mode will no longer be 273 discussed in the following part.

The most unstable coupled Rossby wave mode occurs at the wavelength around 9900km (Fig. 3b). Its corresponding frequency is -0.7765 per year (Fig. 3a) and thus the period is nearly 8 years, close to that of the observed decadal variability. Figure 4 shows the spatial phase relationships between the SST anomaly (shaded) and the upperand lower-layer atmospheric streamfunction as well as upper-oceanic streamfunction anomalies (contours) for the most unstable coupled mode. It can be found that the upper- and lower-layer atmospheric streamfunction anomalies are both in phase with the SST anomaly, indicating that the atmospheric response to the SST anomaly is equivalent barotropic with highs (lows) over warm (cold) water (Fig. 4a and 4b). In

283 addition, the wind-driven upper oceanic streamfunction anomaly has a small eastward 284 phase shift relative to SST and atmospheric streamfunction anomalies, mainly due to the advection process. Such configuration relationship between atmospheric and oceanic anomalies is also consistent with that of the observed decadal variability. Hence,

287 it is suggested that midlatitude ocean-atmosphere interaction through thermal and 288 transient eddy dynamical feedback can produce a coupled oceanic Rossby wave mode 289 on decadal time scale with equivalent barotropic cold trough/warm ridge structure.

Compared with the results of CYF2020 in which the atmosphere is assumed to be 291 barotropic, the period of the most unstable mode becomes longer, and the associated 292 anomalous SST and atmospheric streamfunction are exactly in phase like observation 293 instead of having a small phase shift in $x$ direction (Fig.5). However, the unstable 294 growth rate is reduced by more than half (Fig. 11a). The differences between the two 295 works and the causing reasons will be discussed in detail in Section 5. 


\subsection{Role of diabatic heating with different vertical profiles}

298 In this coupled model, diabatic heat forcing and atmospheric transient eddy vorticity 299 forcing are the two PV sources that jointly affect the seasonal-mean atmospheric 300 circulation anomaly. To examine the relative contributions of different PV sources in 301 destabilizing the coupled oceanic Rossby wave mode, the transient eddy vorticity 302 forcing term is firstly removed by setting $\gamma=0$ in equations (9) and (10). Three cases 303 with different heating profiles are investigated, respectively: surface heating only case $\left(Q_{s} \neq 0, Q=0\right)$, mid-level heating only case $\left(Q_{s}=0, Q \neq 0\right)$ and both surface and 305 mid-level heating case $\left(Q_{s}, Q \neq 0\right)$.

The dispersion relation curves of all the three cases are basically the same as 307 shown in Fig. 6a, 6c and 6e, coinciding with that of the free oceanic Rossby wave. 308 When the surface heating is considered alone, the coupled oceanic Rossby mode also 309 destabilizes over the entire wavelength range (Fig. 6b), but its growth rate decreases 310 significantly compared with the standard case (full PV sources case, Fig. 3b) and the 311 most unstable mode shifts to shorter wavelength at around $8500 \mathrm{~km}$. However, if the 312 mid-level heating is added alone, the coupled oceanic Rossby mode becomes a weak313 damping mode. Comparing the phase relationships of atmospheric and oceanic 314 variables for the two cases (Fig. 7), we can find that the atmospheric responses for the 315 two cases are nearly opposite. To surface cooling induced by cold SST anomaly, the 316 atmospheric response shows an equivalent barotropic lows above cold water anomaly 317 (Fig. 7a and c), similar as in the standard case but with a eastward phase shift relative 318 to SST anomalies. The surface low pressure can sustain the cyclonic oceanic flow and 319 enhance the cold SST anomaly by transporting cold water (Fig. 7e), forming a positive feedback. Thus the stimulated coupled Rossby wave mode is a growing mode. Whereas

321 to the SST induced mid-level cooling, the upper atmospheric streamfunction has an in- 
phase high anomaly response (Fig. $7 \mathrm{~b}$ ), and the lower atmospheric streamfunction anomaly leads that of the upper layer by nearly $\pi / 2$ phase (Fig. $7 d$ ). The surface high pressure will weaken the cyclonic oceanic flow and thus reduce the cold SST anomaly. Therefore, the coupled Rossby wave mode decays gradually.

When the surface and mid-level heating are both included, the results are very similar to that of the surface heating only case (Fig. 6f), indicating that the contribution of diabatic heating to the unstable air-sea coupled mode is dominant by the surface heating. Mid-level heating that tends to cause baroclinic atmospheric response is not

330 conducive to the unstable coupled mode while surface heating always benefits the 331 instability. Figure 10a shows the variation of unstable growth rate with zonal 332 wavelength and surface heating intensity. Here the mid-level heating is fixed and the surface heating is modified continuously by changing $\gamma_{2}$. It can be seen that corresponding to the fastest-growing mode, the stronger the surface heating is (the

335 larger $\gamma_{2}$ is), the more unstable the coupled mode becomes.

\subsection{Role of transient eddy vorticity forcing with different vertical profiles}

337 Similarly, by setting $\gamma_{a}=0$ in equations (9) and (10), the diabatic heating is neglected. 338 Transient eddy vorticity forcing becomes the only PV source of atmosphere. Here we 339 also illustrate three cases with different vertical profiles of transient eddy vorticity 340 forcing: when the vorticity forcing occurs only in lower troposphere $\left(F_{\text {eddy2 }} \neq\right.$ $\left.3410, F_{e d d y 1}=0\right)$, only in higher troposphere $\left(F_{e d d y 2}=0, F_{e d d y 1} \neq 0\right)$, and in both layers $342\left(F_{\text {eddy2 }}, F_{\text {eddy1 } 1} \neq 0\right)$.

343 In all the three cases, the oceanic Rossby wave mode can be unstable over the 344 entire wavelength range (Fig. 8). However, in the lower-layer forcing case, the 345 instability of the coupled mode is very weak. The growth rate of the most unstable mode 346 is only $0.0226\left(\mathrm{yrs}^{-1}\right)$, and the corresponding wavelength and period are $8800 \mathrm{~km}$ 347 and 7.67 yrs, respectively (Fig. 8b and e). While in the higher-layer forcing case, the 
growth rate of the most unstable mode is increased evidently, corresponding to a longer wavelength 9900km and a longer period $8.17 \mathrm{yrs}$ (Fig. 8a and d). Meanwhile, the spatial

350 phase relationship between atmospheric streamfunction and SST anomaly (Fig. 9a and

$351 \mathrm{c}$ ) is closer to that of the standard case (Fig. 3a and b). When the transient eddy vorticity 352 forcing at both layers are considered at the same time, the variation of growth rate is 353 similar as that of the upper-layer forcing case, but the most unstable growth rate is 354 increased slightly with zonal wavelength around 10200km and period of $8.34 \mathrm{yrs}$ (Fig. $3558 \mathrm{c}$ and $\mathrm{f}$ ). Therefore, the upper-layer transient eddy vorticity forcing plays a dominant 356 role in producing unstable air-sea interaction mode.

However, it should be mentioned that in present study, the lower-layer transient eddy vorticity forcing is set to be 0.2 times of that of upper layer $\left(\delta_{2}=0.2\right)$ according to the observational analysis. If we assume the magnitudes of the forcing at both layers are equal $\left(\delta_{2}=1.0\right)$, which is equivalent to the setting in the atmospheric barotropic 361 model (CYF2020), then the unstable growth rate is enhanced greatly no matter whether 362 the higher-layer forcing is considered or not (as shown by the green lines in Fig. 8e and 363 f). At this time, the contribution of individual lower-layer vorticity forcing on the 364 unstable mode has overtook that of individual higher-layer forcing (Fig. 8d and e), suggesting that the lower-layer vorticity forcing stimulates instability more effectively.

366 This can also be illustrated by Fig. 10b, in which the higher-layer forcing is fixed and 367 the lower-layer forcing is changed continuously. With the increase of low-layer forcing, 368 the unstable growth rate increases, and the fastest-growing mode moves to longer 369 wavelength slightly. This phenomenon can also be explained by equations (7) and (8). 370 According to the relationship between transient eddy vorticity forcing and SST 371 anomalies, a cold SST anomaly will cause positive vorticity forcing in both layers. To 372 the barotropic component of the atmosphere, $F_{\text {eddy2 }}$ has equal contribution with $373 F_{\text {eddy } 1}$. But to the baroclinic component, $F_{\text {eddy } 2}$ tends to decrease the baroclinic 374 component by offsetting the contribution of $F_{\text {eddy1 } 1}$. Hence the lower-layer 
atmospheric response of geopotential low anomaly will become stronger and strengthens the cold SST anomaly more effectively through driving cyclonic oceanic flow.

Furthermore, although either diabatic heat forcing or transient eddy vorticity forcing can lead to unstable oceanic Rossby wave mode with similar equivalent barotropic structure, the latter has a much more contribution to the unstable growth rate (Fig.3b, 6f, 8f). It demonstrates that transient eddy dynamic feedback plays a more important role in unstable midlatitude ocean-atmosphere interaction. This conclusion is also consistent with that of previous theoretical study (Fang and Yang, 2016) and analytical model study with barotropic atmospheric model (CYF2020).

\section{Comparison with the results of coupled model with barotropic atmosphere}

In CYF2020, the atmosphere is assumed to be barotropic, and the diabatic heating only occurs at the top of boundary layer. Thus both the diabatic heat forcing that is proportional to the vertical gradient of diabatic heating and the transient eddy vorticity forcing are constant in the vertical direction. Nevertheless, as expressed by equations (7) and (8), in our present baroclinic atmospheric model, the surface diabatic heating only acts on lower-layer atmosphere whereas the mid-level heating impacts on both lower- and higher-layers but with opposite effect. Thus they both contribute to the baroclinic component and their contributions can offset each other to some extent. Moreover, if the transient eddy vorticity forcing is unchanged in vertical direction $\left(F_{e d d y 1}=F_{\text {eddy } 2}\right)$, like in the barotropic model, it will only impact on the barotropic atmospheric component. Otherwise, the difference between the transient eddy vorticity forcing in the two layers will cause baroclinic atmospheric response.

Therefore the barotropic atmosphere model is equivalent to the barotropic component of atmosphere in our study if $U$ and $F_{\text {eddy }}$ are constant. Compared with the results of barotropic mode (orange line in Fig. 11a), the growth rate of the most 
401 unstable oceanic Rossby wave (blue line in Fig. 11a) iss reduced by 70\%. One reason 402 is due to the offset effect by the mid-level diabatic heating (Fig. 11b), and the other 403 reason is due to the weakening of lower-layer transient eddy vorticity forcing (Fig. 11c).

404 As mentioned in Section 3, because the vertical structures of atmospheric PV sources 405 and basic flow are more realistic in the present baroclinic model, the associated period 406 and spatial configuration patterns of the unstable coupled mode approach the 407 observation much better.

\section{Contributions of other processes and factors}

\subsection{Relative roles of oceanic entrainment and advection processes}

In SST evolution equation (12), the oceanic processes determining SST change include

411 horizontal advection, vertical entrainment and air-sea flux exchange, which represent 412 the oceanic adjustment to the atmospheric forcing. The relative roles of different 413 oceanic processes in destabilizing coupled oceanic Rossby wave mode are also 414 examined below.

415 Since the air-sea flux exchange just can stimulates very weak damping mode 416 (figure not shown), the main processes we emphasize here are entrainment and 417 advection. Figure 12 shows the growth rate as a function of wavelength for the coupled 418 oceanic Rossby wave mode for the cases of all oceanic processes, of entrainment only 419 and of advection only, respectively. It can be found that both entrainment and advection 420 have contributions to unstable coupled mode. When the wavelength is shorter than $42110000 \mathrm{~km}$, the total unstable growth is contributed primarily by advection, while over 422 the wavelength range beyond $10000 \mathrm{~km}$, it is contributed jointly by advection and 423 entrainment.

\subsection{Influence of coupling strength and basic zonal flows}

425 The unstable coupled mode may be influenced by two kinds of critical parameters, the 426 coupling coefficients $\left(\gamma_{a}\right.$ and $\gamma$ ) that determining the coupling strength between ocean 
and atmosphere through thermal and dynamical processes, and the background zonal wind in the two atmospheric layers $\left(U_{1}\right.$ and $\left.U_{2}\right)$. For our standard case, the values of these parameters are set as $\gamma=11.2 \mathrm{~s}^{-2} \mathrm{~m}^{2} K^{-1}, \gamma_{a}=8 \times 10^{-7} \mathrm{~s}^{-1}, U_{1}=17 \mathrm{~m} / \mathrm{s}$, $U_{2}=8 \mathrm{~m} / \mathrm{s}$, respectively. The influence of air-sea coupling strength and basic zonal flows on unstable ocean-atmosphere interaction is then estimated by changing the relevant parameter alone.

Figure 13 shows the variation of growth rate of the most unstable Rossby wave mode with zonal wavelength versus dynamical coupling coefficient $(\gamma)$, thermal coupling coefficient $\left(\gamma_{a}\right)$, higher-layer basic zonal wind $\left(U_{1}\right)$, lower-layer basic zonal wind $\left(U_{2}\right)$, and vertical wind shear $\left(\widetilde{U}=U_{1}-U_{2}\right)$, respectively. The standard case is marked by " $X$ " in the figure. It can be seen from Fig. 13a and $b$ that the oceanic Rossby wave is always unstable within the present parameter field. With the increase of coupling strength, no matter dynamical coupling or thermal coupling, the instability of the coupled mode is enhanced. However, the influence of transient eddy dynamical feedback intensity is more prominent, because the enhancement amplitude is larger and the corresponding wavelength range is much wider. When the coupling is strengthened, the SST anomaly can force the atmosphere more strongly, especially through transient eddy vorticity forcing, thus the coupled mode becomes more unstable.

The intensity as well as vertical shear of the basic zonal wind is also a crucial factor that determining the vertical structure of atmospheric response to SST anomalies. When the basic zonal wind in higher layer is larger than that in lower layer $\left(U_{1}>\right.$ $8 \mathrm{~m} / \mathrm{s}$ ), the unstable growth rate decreases with the increase of $U_{1}$ (Fig. 13c). Conversely, when the basic zonal wind in lower layer is less than that in higher layer $\left(U_{2}<17 \mathrm{~m} / \mathrm{s}\right)$, the unstable growth rate increases with the increase of $U_{2}$ (Fig.13d). That is to say, the larger the vertical wind shear, the weaker the instability (Fig. 13e). When the basic zonal winds in both layers are equal, as in the barotropic model, the unstable growth rate reaches its maximum (Fig. 13e). That is because the vertical wind 
454 shear tends to weaken the barotropic atmospheric response by vorticity advection and 455 thus unbenefited to the unstable coupled mode.

\section{Conclusions and discussion}

457 Midlatitude ocean-atmosphere interaction is believed to be an important source of 458 decadal to interdecadal climate variabilities. However, mechanism responsible for the 459 midlatitude ocean-atmosphere interaction remains unclear for a long time primarily due to the lack of understanding on how midlatitude oceans affect the atmosphere.

Traditionally, people think the midlatitude SST impacts on the atmosphere mainly through diabatic heating, as in the tropics. But the pure thermal-driven circulation cannot explain the observed equivalent barotropic structure of decadal variabilities well.

464 Meanwhile, the midlatitude atmosphere has strong baroclinicity, and the synoptic transient eddies accompanied with jet stream and storm track develop vigorously. The thermal and momentum transportation by transient eddies are also important forcing sources of midlatitude atmospheric circulation. Fang and Yang (2016) illustrated that SST anomaly could cause transient eddy forcing anomaly by changing the strength of oceanic front and thus the low-level atmospheric baroclinicity. The atmospheric response to the transient eddy vorticity forcing that dominants the total forcing, displays an equivalent barotropic structure, which further sustains the initial SST anomaly. Hence, transient eddy feedback in the middle latitudes, especially transient eddy dynamic feedback, has been recognized as another important approach for SST to affect the seasonal-mean atmosphere.

In our previous work (CYF2020), an analytical air-sea coupled model is established to investigate the contributions of TEDF to unstable ocean-atmosphere interaction in the middle latitudes. In the model, the atmosphere is assumed to be barotropic for zero-order approximation. In fact, since the midlatitude atmospheric stratification is stable, the diabatic heating is mainly confined to the lower troposphere, 
and there is also a relatively weak heating center in the middle level of atmosphere.

481 Meanwhile, though the transient eddy vorticity forcing has barotropic structure in the 482 vertical direction, its large values primarily locates in middle to high troposphere. In 483 order to resolve the realistic vertical structure of the atmospheric forcing better and 484 further analyze their roles in midlatitude ocean-atmosphere interaction, in the present study, we extend the atmospheric component of the air-sea coupled model into a twolayer baroclinic model.

Both the atmospheric and oceanic components satisfy quasi-geostrophic dynamics and are governed by their QGPV equations on a midlatitude beta plane, respectively. A linearized mixed layer temperature equation is considered to describe the SST evolution. In the coupled model, the ocean is driven by the wind stress forcing of atmosphere and force the atmosphere in turn though diabatic heating and transient eddy vorticity forcing. The diabatic heating is specified at both the top of boundary layer and the interface of two layers, and the diabatic heat forcing in each atmospheric layer is proportional to 494 the vertical gradient of diabatic heating. The transient eddy vorticity forcing in each layer is parameterized with the meridional second derivate of SST. Based on observation, the surface heating is set to be twice the mid-level heating, and the transient eddy vorticity forcing in lower layer is 0.2 times of that in higher layer for the standard case.

Midlatitude air-sea interaction produces two types of coupled modes, in which the oceanic Rossby wave mode that propagates westward destabilizes over the whole range 501 of zonal wavelengths. The fastest-growing oceanic Rossby wave mode occurs at the 502 wavelength around $9900 \mathrm{~km}$, with a period of nearly 8yrs. For this mode, the 503 atmospheric streamfunction response in the two layers are both in phase with SST 504 anomaly, with lows (highs) over cold (warm) water, and the oceanic streamfuction also 505 shows a cyclonic (anticyclonic) circulation anomaly eastward slightly to the cold (warm) 506 SST anomaly. Thus the unstable coupled mode exhibits an equivalent barotropic 
structure similar to the observed decadal climate variability. Such configuration structure also suggests the following air-sea interaction processes, as illustrated in Fig.

509 14. If there is an initial cold (warm) SST anomaly in the north (south), on the one hand, 510 it induces strong surface cooling (warming) and weak mid-level cooling (warming), 511 leading to positive (negative) diabatic heat forcing in both layers. On the other hand, 512 the oceanic front is enhanced by the increase of meridional SST gradient, and so is the 513 low-level atmospheric baroclinicity. The transient eddies become more active and 514 transport more vorticity flux to the north, causing positive (negative) transient eddy 515 vorticity forcing in the north (south) part for both layers. Therefore, through thermal 516 and transient eddy dynamical forcing, the cold (warm) SST anomaly generates an in517 phase atmospheric low (high) anomaly in each atmospheric layer. The surface low (high) pressure anomaly tends to sustain the cyclonic (anticyclonic) oceanic upper flow, and

519 thus further develops the initial cold (warm) SST anomaly through oceanic advection 520 and entrainment processes. Consequently, the amplitude of coupled oceanic Rossby 521 wave grows due to the ocean-atmosphere interaction. vertical profiles in destabilizing the coupled oceanic Rossby wave are further analyzed.

524 A SST anomaly can induce the same-sign surface and mid-level diabatic heating as well 525 as opposite-sign transient eddy vorticity forcing in both layers of the atmosphere. The 526 surface diabatic heating favors the barotropic component of atmosphere and restrains 527 the baroclinic component. Hence it tends to cause barotropic atmospheric response and 528 lead to unstable coupled mode. However, the mid-level heating always enhances the 529 baroclinic component and thus decays the coupled mode by offsetting the effect of 530 surface heating. The contribution of total diabatic heating on the coupled mode is 531 dominated by surface heating. On the other hand, the transient eddy vorticity forcing of 532 each layer can force barotropic atmospheric response and induce unstable coupled 533 mode, while the lower-layer vorticity forcing can affect the unstable mode more 
534 effectively by weakening the baroclinic response. However, in the standard case, the 535 transient eddy vorticity forcing in lower layer is much smaller than that in higher layer, 536 thus the higher-layer transient eddy vorticity forcing has more prominent contribution 537 to the unstable growth rate. Although either diabatic heat forcing or transient eddy 538 vorticity forcing can generate unstable coupled oceanic Rossby wave alone, the 539 transient eddy dynamic feedback plays a much more important role.

540 Besides the SST-induced forcing on the atmosphere, the oceanic adjustment 541 processes driven by the atmospheric circulation, which mainly include advection and 542 entrainment, can also contribute to the unstable coupled mode. When SST is adjusted 543 by advection process alone, the coupled oceanic Rossby wave with zonal wavelength 544 shorter than $10000 \mathrm{~km}$ tends to be unstable. When the entrainment process is considered 545 alone, the unstable coupled mode moves to longer wavelength range $(>10000 \mathrm{~km})$. 546 Moreover, the influence of air-sea coupling strength and background zonal wind are 547 also examined. When the coupling is strengthened, either dynamical coupling or 548 thermal coupling, the instability of the coupled mode is enhanced. But the effect of 549 dynamic coupling through transient eddy vorticity forcing is more significant. For the 550 basic zonal wind, its barotropic part favors the growth of coupled mode, whereas its 551 baroclinic part that determined by the vertical wind shear tends to decay the coupled 552 mode.

553 Compared with the results derived from CYF2020, in which the atmospheric 554 component is barotropic, the spatial configuration structure and period of the unstable 555 coupled mode are more consistent with the observation. Nevertheless, it should be 556 mentioned that there are actually three PV sources in the atmospheric QGPV equation: 557 diabatic heat forcing, transient eddy heat forcing and transient eddy vorticity forcing 558 (Fang and Yang, 2016). Transient eddy heat forcing is proportional to the vertical 559 gradient of transient eddy heating $\left(Q_{e d d y}\right)$ that represents the convergence/divergence 560 of heat flux transported by transient eddies. Transient eddy heating that has the same 
561 dimension of diabatic heating can be seen as an indirect heat source of the atmosphere, 562 reflecting the transient eddy thermal forcing on the atmosphere. The magnitude of 563 transient eddy heating is comparable with that of diabatic heating, and it mainly 564 concentrates in the middle of troposphere (Fig. 2c in CYF2020). Hence in the barotropic 565 model, it is neglected after vertical integration. In the present study, there is still on 566 explicit performance of transient eddy heating in the coupled model. However, since it 567 is equivalent to a mid-level heating, the case with equal diabatic heating at surface and 568 middle level of atmosphere $\left(\gamma_{2}=1\right.$ in Fig. 10a) in this study can represent the 569 condition in which the transient eddy heating is also considered in the coupled model. 570 At this time, the growth rate of the most unstable coupled mode is decreased a little due 571 to the enhancement of baroclinic response induced by transient eddy heating.

572 In addition, according to the form of plane wave solutions in our study, all the 573 variables are required to be in phase in $y$ direction. Thus the meridional phase shift 574 as shown in Fig.5c and d cannot be obtained. If we use another form of plane wave

575 solutions as $\left(\psi_{a}, \psi_{o}, T_{1}\right) e^{i(k x+l y-\sigma t)}$, there will not be closed spatial centers for the 576 perturbation, but the meridional phase shift between SST and atmospheric 577 streamfunction as well as oceanic streamfunction can be exhibited.

\section{Acknowledgements}

579 This study is jointly supported by the National Natural Science Foundation of China 580 (Grant Nos. 41621005 and 41875086) and the National Key Basic Research and 581 Development Program of China (Grant No. 2018YFC1505902). We are grateful for the 582 support from the Jiangsu Collaborative Innovation Center for Climate Change. 


\section{References}

Cayan DR (1992) Latent and sensible heat flux anomalies over the northern oceans: Driving the sea surface temperature. J Phys Oceanogr 22: 859-881. https://doi.org/10.1175/1520-0485(1992)022<0859:LASHFA>2.0.CO;2

Chen L Chen, L., Fang, J. \& Yang, X. Midlatitude unstable air-sea interaction with atmospheric transient eddy dynamical forcing in an analytical coupled model. Clim Dyn (2020). https://doi.org/10.1007/s00382-020-05405-0

Chu CJ, Yang XQ, Ren XJ, Zhou TJ (2013) Response of Northern Hemisphere storm tracks to Indian-western Pacific Ocean warming in atmospheric general circulation models. Clim Dyn 40(5-6):1057-1070. https://doi.org/10.1007/s00382-013-1687-y

Deser C, Blackman M (1993) Surface climate variations over the North Atlantic Ocean during winter: 1900-1989. J Clim 6:1743-1753. https://doi.org/10.1175/1520-0442(1993)006<1743:SCVOTN>2.0.CO;2

Enfield DB, Mestas-Nunez AM (1999) Multiscale variabilities in global sea surface temperatures and their relationships with tropospheric climate patterns. J Clim 12:2719-2733. https://doi.org/10.1175/1520-0442(1999)0122.0.CO;2

Fang J, Yang X-Q (2011) The relative roles of different physical processes in unstable midlatitude ocean-atmosphere interactions. J Clim, 24: 1542-1558. https://doi.org/10.1175/2010JCLI3618.1

Fang J, Yang X-Q (2016) Structure and dynamics of decadal anomalies in the wintertime midlatitude North Pacific ocean-atmosphere system. Clim Dyn 47: 1989-2007. https://doi.org/10.1007/s00382-015-2946-X

Fang JB, Rong XY, Yang XQ (2006) Decadal-to-interdecadal response and adjustment of the North Pacific to prescribed surface forcing in an oceanic general circulation model. Acta Oceanol Sin 25(3):11-24. https://doi.org/10.1016/j.marchem.2005.09.003 
612 Graham NE, Barnett TP, Wilde R, Ponater M, Schubert S (1994) On the roles of 613 tropical and midlatitude SSTs in forcing interannua to interdecadal variability in 614 the winter Northern Hemisphere circulation. J Clim 7:1416-1441.

615 https://doi.org/10.1175/1520-0442(1994)007<1416:OTROTA>2.0.CO;2

616 Gu D, Philander SGH (1997) Interdecadal climate fluctuations that depend on

617 exchange between the tropics and extratropics. Science 275:805-807.

$618 \quad$ https://doi.org/10.1126/science.275.5301.805

619 Hoskins BJ, and DJ Karoly (1981) The steady linear response of a spherical

620 atmosphere to thermal and orographic forcing. J. Atmos. Sci., 38, 1179-1196.

621 http://dx.doi.org/10.1175/1520-0469(1981)038<1179:TSLROA>2.0.CO;2

622 Kushnir Y, Robinson WA, Bladé I, Hall NMJ, Peng S, Sutton R (2002)Atmospheric

623 GCM response to extratropical SST anomalies: Synthesis and evaluation. J Clim

624 15:2233-2256. https://doi.org/10.1175/1520-

$625 \quad$ 0442(2002)015<2233:AGRTES>2.0.CO;2

626 Latif M, Barnett TP (1994) Causes of decadal climate variability over the North

627 Pacific and North America. Science 266:634-637.

$628 \quad$ https://doi.org/10.1126/science.266.5185.634

629 Latif M, Barnett TP (1996) Decadal climate variability over the North Pacific and

630 North America: dynamics and predictability. J Atmos Sci 48: 2589-2631.

631 https://doi.org/10.1175/1520-0442(1996)009<2407:DCVOTN>2.0.CO;2

632 Latif M, 1999: Dynamics of interdecadal variability in coupled ocean-atmosphere

633 models. Beyond El Nino: Decadal and Interdecadal Climate Variability. A.

634 Navarra, Ed., Springer Verlag, 213-250. https://doi.org/10.1007/978-3-642-

635 58369-8_11

636 Lau NC, Holopainen EO (1984) Transient eddy forcing of the time-mean flow as

637 identified by geopotential tendencies. J Atmos Sci 41: 313-328.

638 https://doi.org/10.1175/1520-0469(1984)041<0313:TEFOTT>2.0.CO;2 
Liu Z, Wu L (2004) Atmospheric response to North Pacific SST anomaly: the role of ocean-atmosphere coupling. J Clim 17:1859-1892. https://doi.org/10.1175/15200442(2004)017<1859:ARTNPS>2.0.CO;2

Liu CJ, Ren XJ, Yang XQ (2014) Mean flow-storm track relationship and Rossby wave breaking in two types of El-Niño. Adv Atmos Sci 31(1):197-210. https://doi.org/10.1007/s00376-013-2297-7

Mantua NJ, Hare SR, Zhang Y, Wallace JM, Francis RC (1997) A Pacific interdecadal climate oscillation with impacts on salmon production. Bull Am Meteorol Soc 78, 1069-1079. https://doi.org/10.1175/15200477(1997)078<1069:APICOW>2.0.CO;2

Minobe S (1997) A 50-70 year climatic oscillation over the North Pacific and North America. Geophys Res Lett 24: 683-686. https://doi.org/10.1029/97GL00504

Nakamura H, Sampe T, Tanimoto Y, Shimpo A (2004) Observed associations among storm tracks, jet streams and midlatitude oceanic fronts. Earth's climate: the ocean-atmosphere interaction. Geophys. Monogr., vol 147. American Geophysical Union, pp 329-345. https://doi.org/10.1029/147GM18

Namias J, Cayan DR (1981) Large-scale air-sea interactions and short period climate fluctuations. Science 214:869-876. https://doi.org/ 10.1126/science.214.4523.869

Neelin JD, Weng W (1999) Analytical prototypes for ocean-atmosphere interaction at midlatitudes. Part I: Coupled feedbacks as a sea surface temperature dependent stochastic process. J Clim 12: 697-721. https://doi.org/10.10021175/15200442(1999)012<0697:APFOAI>2.0.CO;2

Nie Y, Zhang Y, Yang XQ, Chen G (2013) Baroclinic anomalies associated with the Southern Hemisphere annular mode: roles of synoptic and low-frequency eddies. Geophys Res Lett 40:2361-2366. https://doi.org/10.1002/grl.50396 
664

665

666

667

668

669

670

671

672

673

674

675

676

677

678

679

680

681

682

683

684

685

686

687

688

689

690

691

Nie Y, Zhang Y, Chen G, Yang XQ, Alex Burrows D (2014) Quantifying barotropic and baroclinic eddy feedbacks in the persistence of the Southern Annular Mode. Geophys Res Lett 41:8636-8644.https://doi.org/10.1002/2014GL062210

Pedlosky J (1970) Finite-amplitude baroclinic waves. J Atmos Sci 27: 15-30. https://doi.org/10.1175/1520-0469(1970)027<0015:FABW>2.0.CO;2

Qiu B, Schneider N, Chen S (2007) Coupled decadal variability in the North Pacific: an observationally constrained idealized model. J Clim 20:3602-3620. https://doi.org/10.1175/JCLI4190.1

Qiu S, FANG J-B, YANG X-Q. Mid-Latitude Atmospheric Responses to Heat and Vorticity Using a Linear Baroclinic Model. J Meteor Sci, 2014, 34: 149-161. https://doi.org/10.3969/2013jms.0060

Ren XJ, Yang XQ, Chu CJ (2010) Seasonal variations of the synoptic- scale transient eddy activity and polar-front jet over east Asia. J Clim 23(12):32223233.https://doi.org/10.1175/2009JCLI3225.1

Robertson AW (1996): Interdecadal variability over the North Pacific in a multicentury climate simulation. Clim Dyn 12: 227-241. https://doi.org/10.1007/BF00219498

Saravanan R, McWilliams JC (1997) Stochasticity and spatial resonance in interdecadal climate fluctuations. J Clim 10: 2299-2320. https://doi.org/10.1175/1520-0442(1997)0102.0.CO;2

Saravanan R, McWilliams JC (1998) Advective ocean-atmosphere interaction: An analytical stochastic model with implications for decadal variability. J Clim 11: 165-188. https://doi.org/10.1175/15200442(1998)011<0165:AOAIAA>2.0.CO;2

Shutts G (1987) Some comments on the concept of thermal forcing. Q J R Meteorol Soc 113: 1387-1394. https://doi.org/10.1002/qj.49711347817

Small RJ, deSzoeke SP, Xie SP, O’Neill L, Seo H, Song Q, Cornillon P, Spall M, Minobe S (2008) Air-sea interaction over ocean fronts and eddies. Dyn Atmos 
Tao L, Yang X-Q, Fang J, Sun X (2020) PDO-related wintertime atmospheric anomalies over the midlatitude North Pacific: Local versus remote SST forcing. J Clim. https://doi.org/10.1175/JCLI-D-19-0143.1

Trenberth KE (1990) Recent observed interdecadal climate changes in the Northern Hemisphere. Bull Am Meteorol Soc 71:988-993. https://doi.org/10.1175/15200477(1990)071<0988:ROICCI>2.0.CO;2

Wang L, Yang X-Q Yang, Yang D, Xie Q, Fang J, Sun X (2017) Two typical modes in the variabilities of wintertime North Pacific basin-scale oceanic fronts and associated atmospheric eddy-driven jet. Atmospheric Science Letters, 18: 373380, doi: 10.1002/asl.766.

Wang L, Hu H, Yang X-Q (2019) The atmospheric responses to the intensity variability of subtropical front in the wintertime North Pacific. Clim Dyn 52: 5623-5639. https://doi.org/10.1007/s00382-018-4468-9

Xiang Y, Yang XQ (2012) The effect of transient eddy on interannual meridional displacement of summer east Asian subtropical jet. Adv Atmos Sci 29(3):484492. https://doi.org/10.1007/s00376-011-1113-5

Zhang R, Fang J, and Yang X-Q (2020) What kinds of atmospheric anomalies drive wintertime North Pacific basin-scale subtropical oceanic front intensity variation? Journal of Climate, 33 (16): 7011-7026, doi: 10.1175/JCLI-D-190973.1.

Zhang Y, Yang XQ, Nie Y, Chen G (2012) Annular-mode-like variation in a multilayer QG model. J Atmos Sci 69(10):2940-2958. https://doi.org/10.1175/JAS-D11-0214.1

Zhong YF, Liu Z, Jacob R (2008) Origin of Pacific multi-decadal vari- ability in Community Climate System Model version (CCSM3): a combined statistical and 
dynamical assessment. J Clim 21:114-133. https://doi.org/10.1175/2007JCLI1730.1

Zorita E, and Frankignoul C(1997) Modes of North Atlantic decadal variability in the ECHAM1/LSG coupled ocean-atmosphere general circulation model. J Clim 10: 183-200. https://doi.org/10.1175/15200442(1997)010<0183:MONADV>2.0.CO;2

Zhu YM, Yang XQ (2003) Joint propagating patterns of SST and SLP anomalies in the North Pacific on bidecadal and pentadecadal timescales. Adv Atmos Sci Pacific as simulated by FGOALS-g fast coupled climate model. Chin J Geophys 51(1):58-69. https://doi.org/10.1002/cjg2.1194

730 
Table 1. Standard values of parameters used in the coupled air-sea model

\begin{tabular}{|c|c|c|}
\hline Symbol & Parameter & Value \\
\hline$\overline{f f}$ & Coriolis parameter & $1 \times 10^{-4} s^{-1}$ \\
\hline$\beta$ & Meridional gradient of $f$ & $1.8 \times 10^{-11}(\mathrm{~ms})^{-1}$ \\
\hline$\alpha$ & Wind stress coupling constant & $3 \times 10^{-8} s^{-1}$ \\
\hline$L_{o}$ & Oceanic Rossby radius of deformation & $45 \mathrm{~km}$ \\
\hline$L_{a}$ & $\begin{array}{c}\text { Atmospheric Rossby radius of } \\
\text { deformation }\end{array}$ & $660 \mathrm{~km}$ \\
\hline$l$ & Meridional wavenumber & $2 \pi /(6400 \mathrm{~km})$ \\
\hline$T_{r}$ & Reference temperature of deep layer & $283 K$ \\
\hline $\bar{T}_{1}^{c}$ & Mean sea surface temperature & $293 K$ \\
\hline$N_{a}^{2}$ & $\begin{array}{l}\text { Square of the atmospheric Brunt- } \\
\text { Väisälä buoyancy frequency }\end{array}$ & $4.356 \times 10^{-5} s^{-2}$ \\
\hline$C_{p}$ & $\begin{array}{l}\text { Oceanic specific heat at constant } \\
\text { pressure }\end{array}$ & $4000 \mathrm{Jg}^{-1} \mathrm{~K}^{-1}$ \\
\hline$b$ & Buoyancy of thermocline & $2.5 \times 10^{-2} N$ \\
\hline$U_{1}$ & $\begin{array}{c}\text { Mean zonal wind for atmospheric } \\
\text { layer } 1\end{array}$ & $17 m s^{-1}$ \\
\hline$U_{2}$ & $\begin{array}{l}\text { Mean zonal wind for atmospheric } \\
\text { layer } 2\end{array}$ & $8 m s^{-1}$ \\
\hline$c_{4}$ & $\begin{array}{c}\text { Mean oceanic meridional temperature } \\
\text { gradient }\end{array}$ & $3 \times 10^{-6} K m^{-1}$ \\
\hline$r_{s}$ & Rayleigh damping coefficient & $1.3 \times 10^{-7} s^{-1}$ \\
\hline$r$ & Diffusive coefficient & $1 /(5 d a y)$ \\
\hline$z(h)$ & Height of atmospheric boundary layer & $1200 m$ \\
\hline$H_{a}$ & Depth of free troposphere & $10 \mathrm{~km}$ \\
\hline$H_{1}$ & Mixed layer depth & $100 m$ \\
\hline$H$ & Mean ocean upper-layer depth & $500 m$ \\
\hline $\mathrm{H}_{2}$ & Thermocline layer mean depth & $400 m$ \\
\hline$\Delta h_{e}$ & Entrainment layer depth & $40 m$ \\
\hline$\theta_{a 0}$ & $\begin{array}{l}\text { Typical atmospheric potential } \\
\text { temperature }\end{array}$ & $290 K$ \\
\hline$\gamma_{0}$ & Oceanic air-sea flux parameter & $5 \times 10^{-8} s^{-1}$ \\
\hline$\gamma_{a}$ & $\begin{array}{l}\text { Coupling coefficient for diabatic } \\
\text { heating forcing }\end{array}$ & $8 \times 10^{-7} s^{-1}$ \\
\hline$\gamma$ & $\begin{array}{l}\text { Coupling coefficients for transient } \\
\text { eddy vorticity forcing }\end{array}$ & $11.2 s^{-2} m^{2} K^{-1}$ \\
\hline$\gamma_{2}$ & $\begin{array}{c}\text { Ratio of surface diabatic heating to } \\
\text { mid-level heating }\end{array}$ & 2 \\
\hline$\delta_{2}$ & $\begin{array}{c}\text { Ratio of transient eddy vorticity } \\
\text { forcing in lower layer to that in } \\
\text { higher layer }\end{array}$ & 0.2 \\
\hline
\end{tabular}


734 Figure 1. Schematic diagram of vertical structure of the analytical midlatitude coupled ocean-atmosphere model and corresponding variables.

Figure 2. Latitude-altitude sections of regressed wintertime (DJF) anomalies (shading) upon the standardized PDO index during 1960-2010 for (a) geopotential height (units: $m$ ), (b) diabatic heating (units: $K /$ day), and (c) transient eddy vorticity forcing (units: $10^{-10} \mathrm{~S}^{-2}$ ), averaged between $150^{\circ} \mathrm{E}$ and $150^{\circ} \mathrm{W}$, in which the black contours in (b, c, d) represent their corresponding climatologies, and the dots indicate the regions passing the student $t$-test at $95 \%$ significant level. A corresponding meridional distribution of regressed sea surface temperature anomalies (units: $K$ ) is shown in the lower panel of (a). This figure is quoted from CYF2020 .

\section{Figure 3. Climatological distributions of wintertime (a) SST (units: $K$ ), (b) 2m-air} temperature (units: $K$ ), (c) negative meridional gradient of SST (units: $10^{-5} \mathrm{~K}$. $\mathrm{m}^{-1}$ ), (d) negative meridional gradient of $2 \mathrm{~m}$-air temperature (units: $10^{-5} \mathrm{~K} \cdot \mathrm{m}^{-1}$ ), (e) Eady growth rate at $850 \mathrm{hPa}$ (units: $d a y^{-1}$ ), and (f) zonal wind (units: $\mathrm{m} / \mathrm{s}$ ) at $300 \mathrm{hPa}$ for $1960-2010$.

Figure 4. Spatial phase relationships between the SST anomaly (shaded) and (a) the upper-layer atmospheric streamfunction anomaly, (b) the lower-layer atmospheric streamfunction anomaly and (c) the upper-oceanic streamfunction anomaly (contours) for the fastest-growing coupled oceanic Rossby mode, given a fixed meridional wavelength at $6400 \mathrm{~km}$. The largest amplitude of the SST anomaly is specified as 2K.

Figure 5. Spatial distributions of regressed wintertime anomalies (shading) upon the standardized PDO index during 1960-2010 for (a) SST (units: K), (b) 2m-air temperature (units: $K$ ), (c) negative meridional gradient (units: $10^{-5} \mathrm{~K} \cdot \mathrm{m}^{-1}$ ) of SST, (d) negative meridional gradient (units: $10^{-5} \mathrm{~K} \cdot \mathrm{m}^{-1}$ ) of $2 \mathrm{~m}$-air temperature, (e) Eady growth rate at $850 \mathrm{hPa}$ (units: $d a y^{-1}$ ), and (f) zonal wind (units: $\mathrm{m} / \mathrm{s}$ ) at 
$300 \mathrm{hPa}$. The dots indicate the regions passing the student $t$-test at $95 \%$ significant level.

Figure 6. Frequency (a, c, e) and growth rate (b, d, f) of the coupled oceanic Rossby wave mode as a function of zonal wavelength corresponding to the surface diabatic heating only case $(a, b)$, the mid-level heating only case $(c, d)$ and the both surface and mid-level heating case (e, f), respectively. Red lines in (a, c, e) denote the frequency of free oceanic Rossby wave.

Figure 7. As in Fig. 4, but derived with (left) the surface diabatic heating alone and (right) the mid-level diabatic heating alone.

Figure 8. Frequency (a, b, c) and growth rate (blue lines in d, e, f) of the coupled oceanic Rossby wave mode as a function of zonal wavelength corresponding to cases when the transient eddy vorticity forcing occurs in lower layer only (a, d), in higher layer only (b, e), and in both layers (c, f), respectively. Green lines in (e, f) indicate the associated growth rate when the transient eddy vorticity forcing in lower layer is increased to be equal to that in higher layer. Red lines in (a, c, e) denote the frequency of free oceanic Rossby wave.

Figure 9. Spatial phase relationships among characteristic SST (blue line) atmospheric streamfunction (green lines) and upper-oceanic streamfunction (purple lines) anomalies in (a) $X$ direction and (b) $Y$ direction, respectively, for the fastest growing coupled oceanic Rossby mode. As a comparison, the observed wintertime SST anomalies (shading, units: degree in $K$ ) together with vertical integration of atmospheric streamfunction anomalies (contours, units: $10^{-4} \mathrm{~m}^{2} / \mathrm{s}$ ), and SST anomalies together with upper-oceanic streamfunction anomalies (contours, units: $10^{-4} \mathrm{~m}^{2} / \mathrm{s}$ ), regressed on the standardized PDO index, are shown in (c) and (d), respectively. 
784 Figure 10. Unstable growth rate of coupled oceanic Rossby wave mode as a function 785 of zonal wavelength versus (a) the ratio of surface heating to mid-level heating $\left(\gamma_{2}\right.$, 786 here the mid-level heating is fixed and the surface heating is enhanced with the 787 increase of $\gamma_{2}$ ), and (b) the ratio of lower-layer transient eddy vorticity forcing to 788 higher-layer vorticity forcing $\left(\delta_{2}\right.$, here the higher-layer vorticity forcing is fixed and 789 the lower-layer vorticity forcing is enhanced with the increase of $\delta_{2}$ ), respectively.

790 Figure 11. Comparison of the growth rate of the coupled oceanic Rossby wave mode 791 derived in our study with that derived in CYF2020 when (a) both diabatic heating and 792 transient eddy vorticity forcing are considered simultaneously, (b) the diabatic heating 793 is considered only and (c) the transient eddy vorticity forcing is considered only.

794 Figure 12. Growth rate as a function of wavelength for the coupled oceanic Rossby 795 wave mode with (a) full oceanic processes in SST evolution equation, (b) 796 entrainment- process only, and (c) advection process only. Note that (a) is the same as $797 \quad$ Fig. 3b.

798 Figure 13. Unstable growth rate of coupled oceanic Rossby wave mode as a function 799 of zonal wavelength versus (a) dynamical coupling coefficient $(\gamma)$, (b) thermal 800 coupling coefficient $\left(\gamma_{a}\right)$, (c) the higher-layer background zonal wind $\left(U_{1}\right),(\mathrm{d})$ the 801 lower-layer background zonal wind $\left(U_{2}\right)$ and (e) the vertical wind shear $\left(U_{1}-U_{2}\right)$, 802 respectively.

803 Figure 14. Schematic diagram of the phase relationships among atmospheric and 804 oceanic variables for the most unstable coupled oceanic Rossby wave mode in the 805 midlatitudes. 


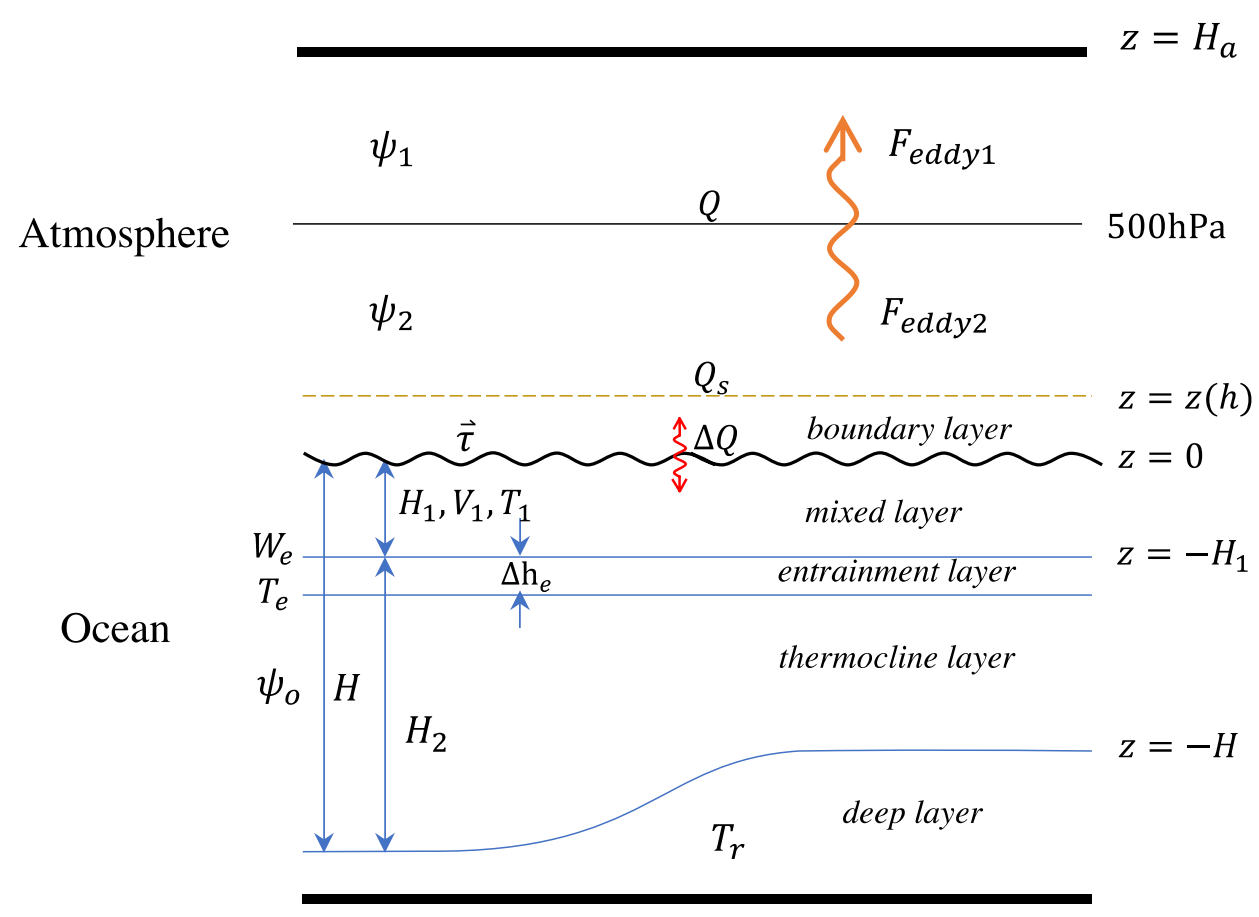

808

809 Figure 1. Schematic diagram of vertical structure of the analytical midlatitude

810 coupled ocean-atmosphere model and corresponding variables.

811 

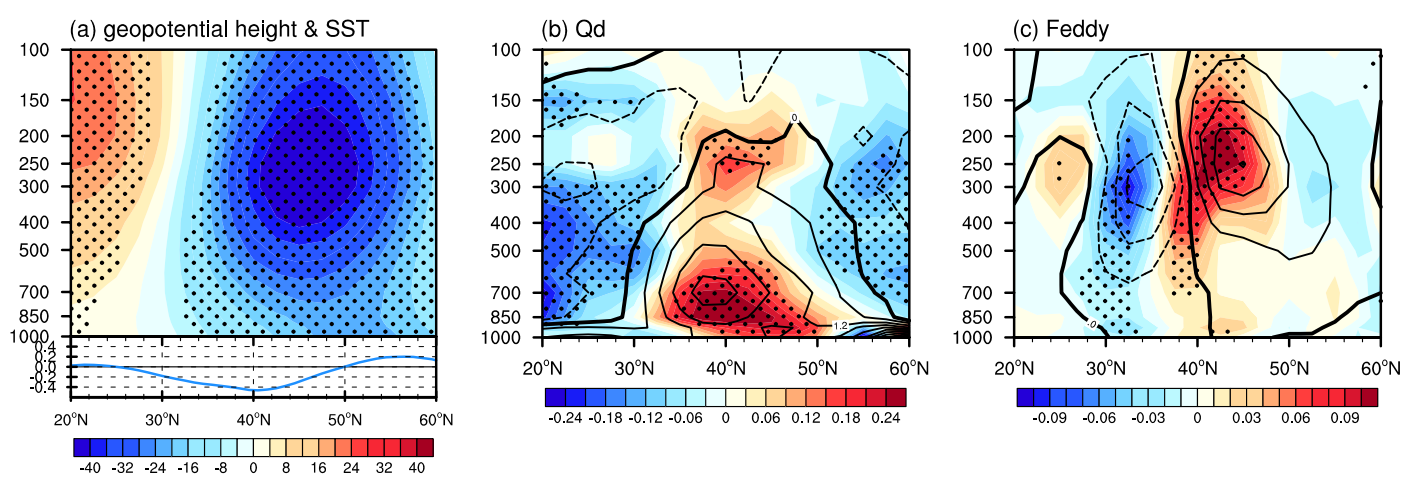

814 Figure 2. Latitude-altitude sections of regressed wintertime (DJF) anomalies

815 (shading) upon the standardized PDO index during 1960-2010 for (a) geopotential

816 height (units: $m$ ), (b) diabatic heating (units: $K /$ day), and (c) transient eddy

817 vorticity forcing (units: $10^{-10} s^{-2}$ ), averaged between $150^{\circ} \mathrm{E}$ and $150^{\circ} \mathrm{W}$, in which

818 the black contours in (b, c, d) represent their corresponding climatologies, and the

819 dots indicate the regions passing the student $t$-test at $95 \%$ significant level. A

820 corresponding meridional distribution of regressed sea surface temperature anomalies

821 (units: $K$ ) is shown in the lower panel of (a). This figure is quoted from CYF2020. 
(a)

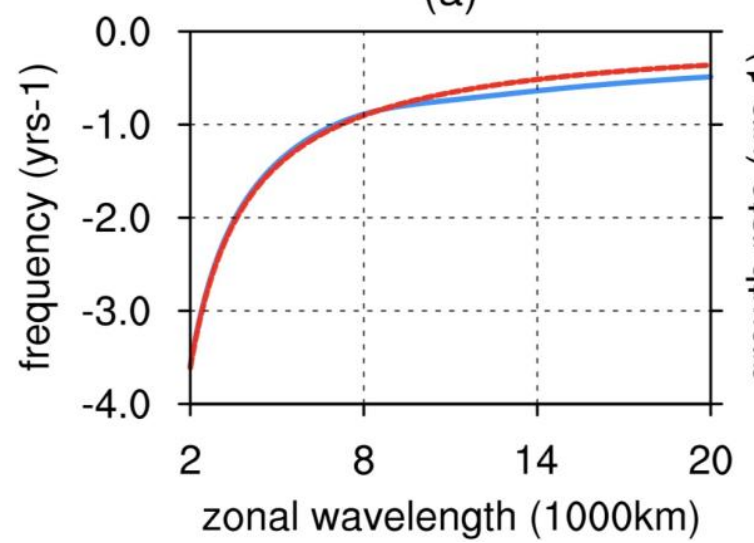

(b)

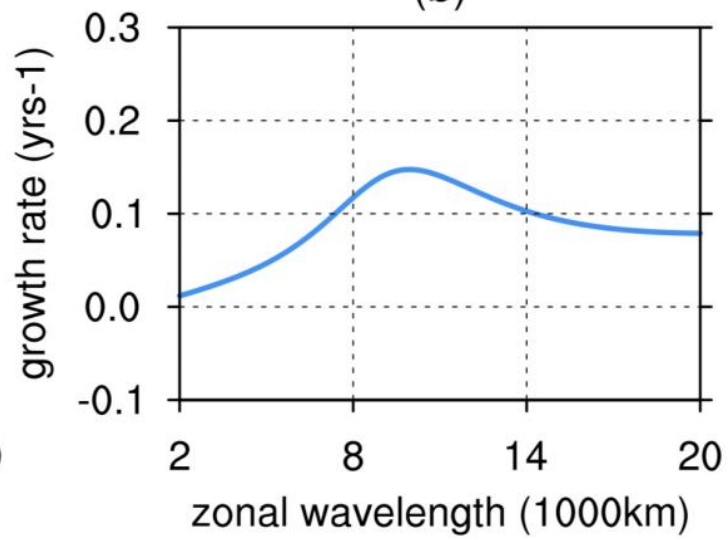

826 Figure 3. (a) Frequency and (b) growth rate of the coupled oceanic Rossby wave 827 mode as a function of zonal wavelength. The red line in (a) denotes the frequency of 828 free oceanic Rossby wave.

829 


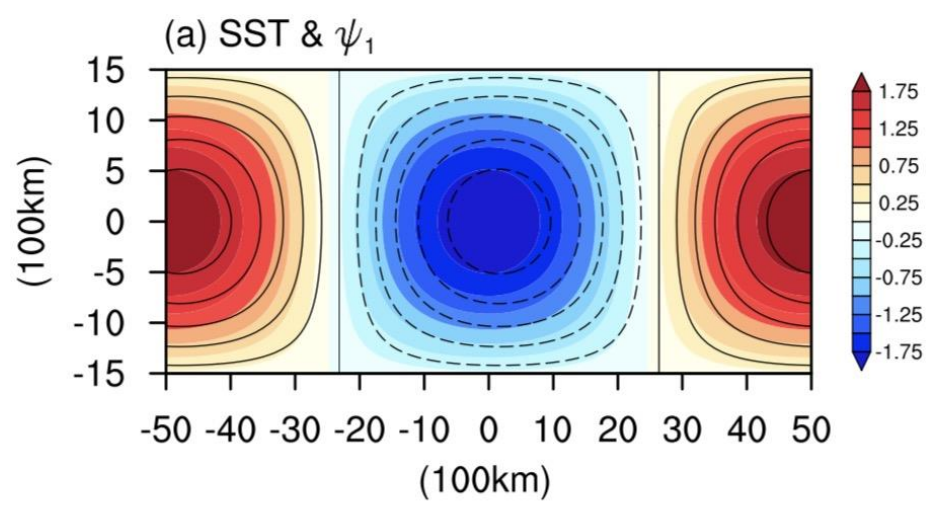

(b) SST \& $\psi_{2}$

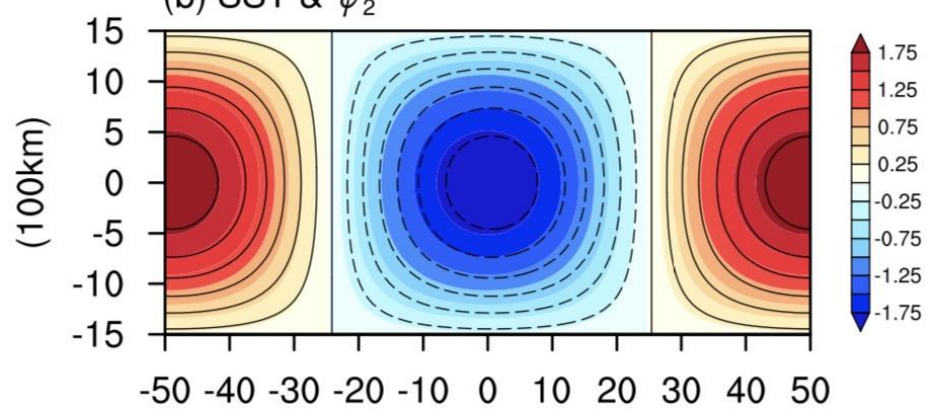

(c) SST \& $\psi_{0}$

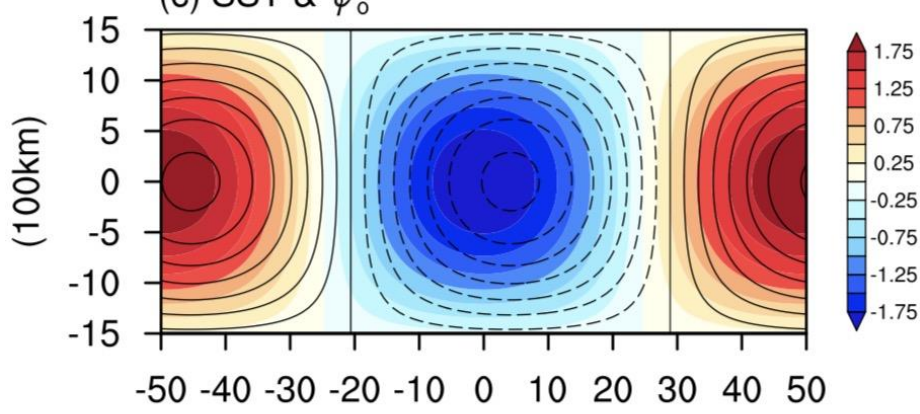

$(100 \mathrm{~km})$

832 Figure 4. Spatial phase relationships between the SST anomaly (shaded) and (a) the

833 upper-layer atmospheric streamfunction anomaly, (b) the lower-layer atmospheric

834 streamfunction anomaly and (c) the upper-oceanic streamfunction anomaly

835 (contours) for the fastest-growing coupled oceanic Rossby mode, given a fixed

836 meridional wavelength at $6400 \mathrm{~km}$. The largest amplitude of the SST anomaly is

837 specified as $2 \mathrm{~K}$. 
(a) SST \& $\psi_{\mathrm{a}}$

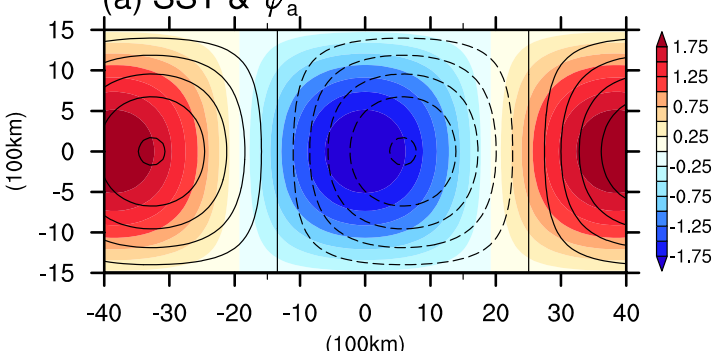

(c) SST \& $\psi_{\mathrm{a}}$ regressed by PDO

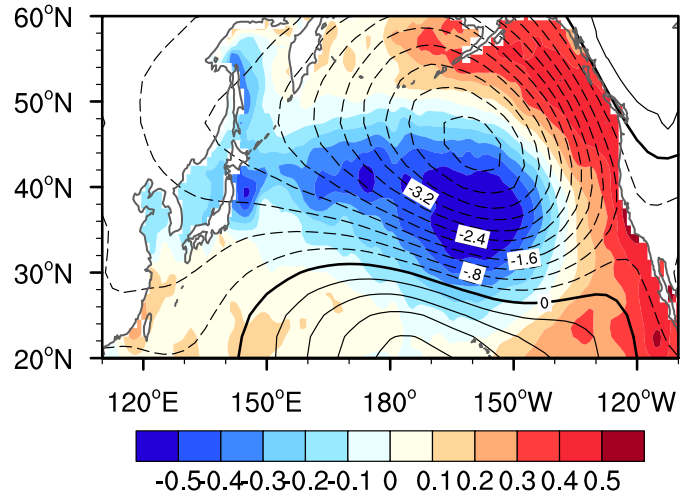

(b) SST \& $\psi_{0}$

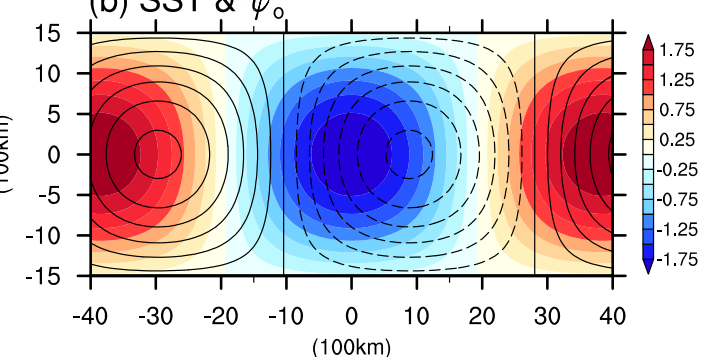

(d) SST \& $\psi_{0}$ regressed by PDO

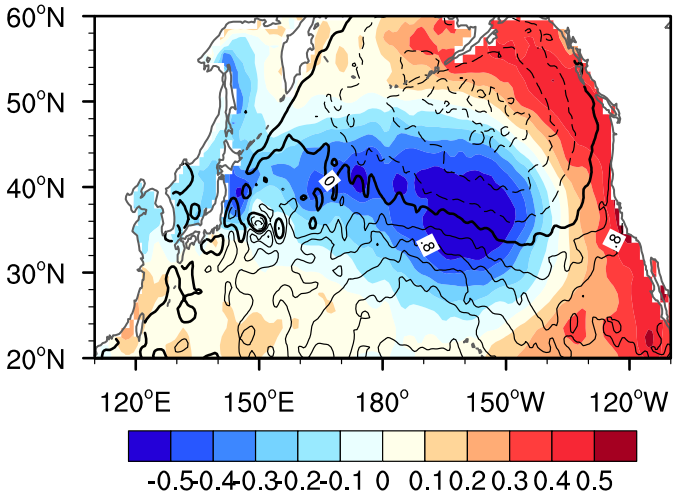

840 Figure 5. Spatial phase relationships between characteristic SST anomaly (shaded)

841 and (a) barotropic atmospheric streamfunction anomaly and (b) upper-oceanic

842 streamfunction anomaly (contours) for the fastest-growing coupled oceanic Rossby

843 mode in CYF2020. The observed wintertime SST anomalies (shaded, units: degree in

$844 K$ ) together with (c) vertical integration of atmospheric streamfunction anomalies

845 (contours, units: $10^{-4} \mathrm{~m}^{2} / \mathrm{s}$ ), and (d) upper-oceanic streamfunction anomalies

846 (contours, units: $10^{-4} \mathrm{~m}^{2} / \mathrm{s}$ ), regressed on the standardized PDO index, are shown in

847 (c) and (d), respectively. This figure is quoted form CYF2020. 
(a) Qs

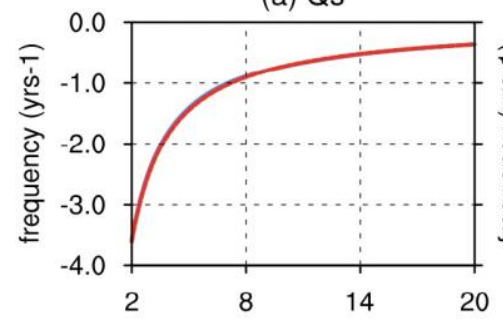

(b) Qs

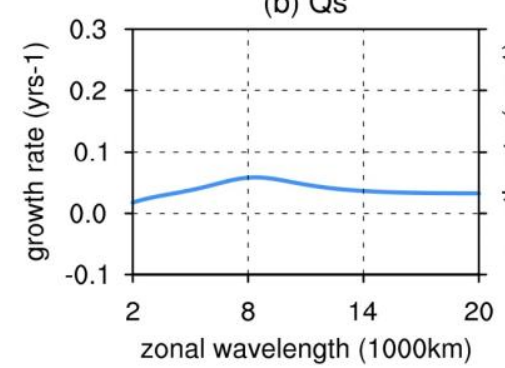

(c) $\mathrm{Q}$

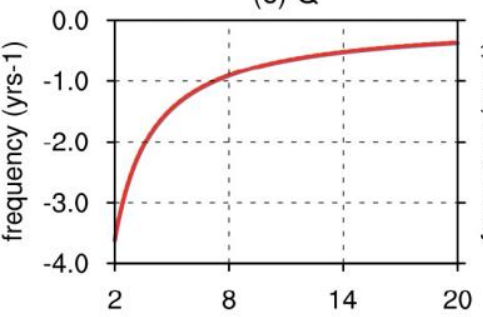

(d) $\mathrm{Q}$

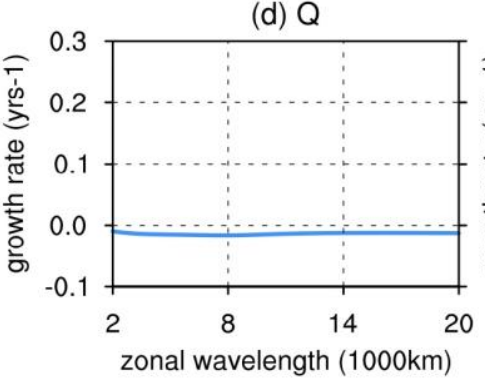

(e) $Q+Q s$

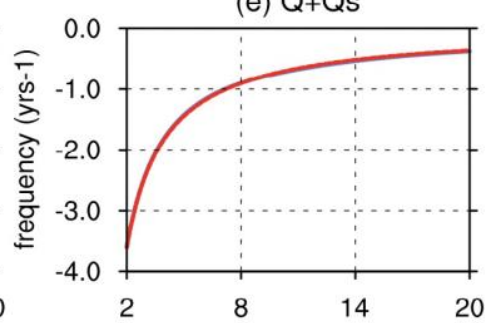

(f) $Q+Q s$

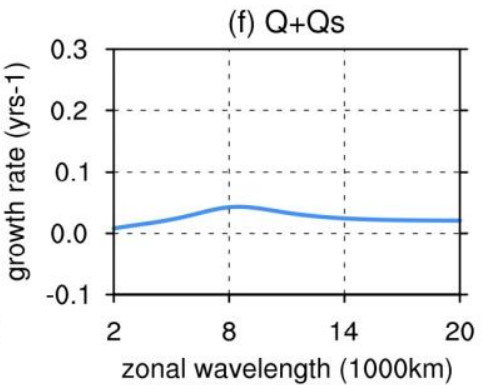

856 Figure 6. Frequency (a, c, e) and growth rate (b, d, f) of the coupled oceanic Rossby

857 wave mode as a function of zonal wavelength corresponding to the surface diabatic

858 heating only case (a, b), the mid-level heating only case(c, d) and the both surface and

859 mid-level heating case (e, f), respectively. Red lines in (a, c, e) denote the frequency

860 of free oceanic Rossby wave. 
862

Qs

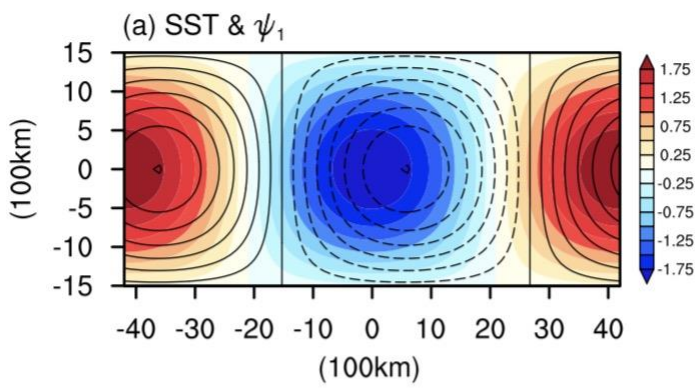

(c) SST \& $\psi_{2}$

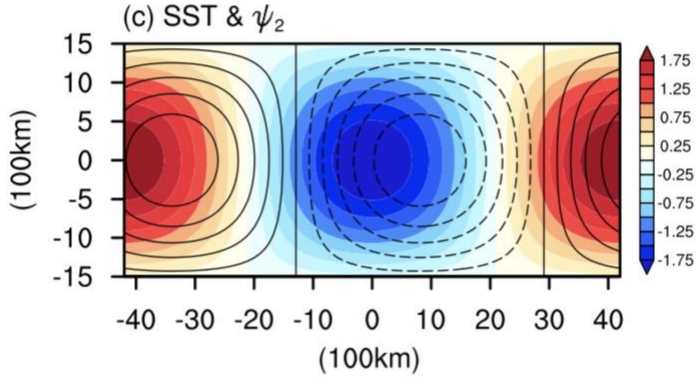

(e) SST \& $\psi_{0}$

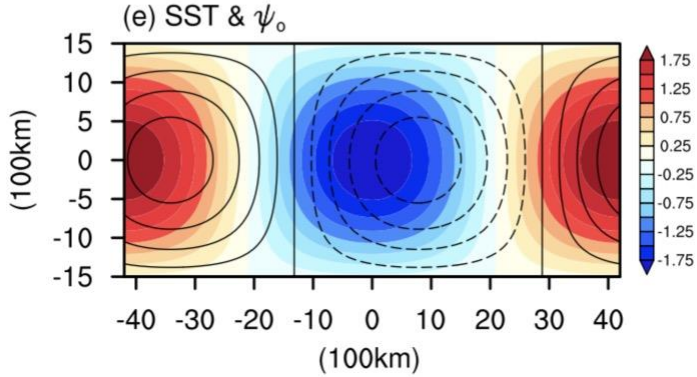

Q

(b) SST \& $\psi_{1}$

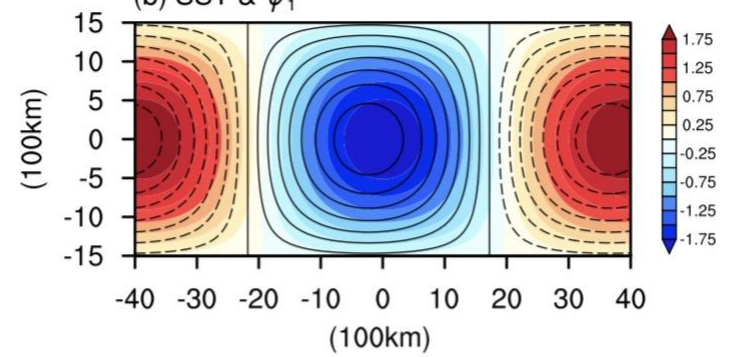

(d) SST \& $\psi_{2}$
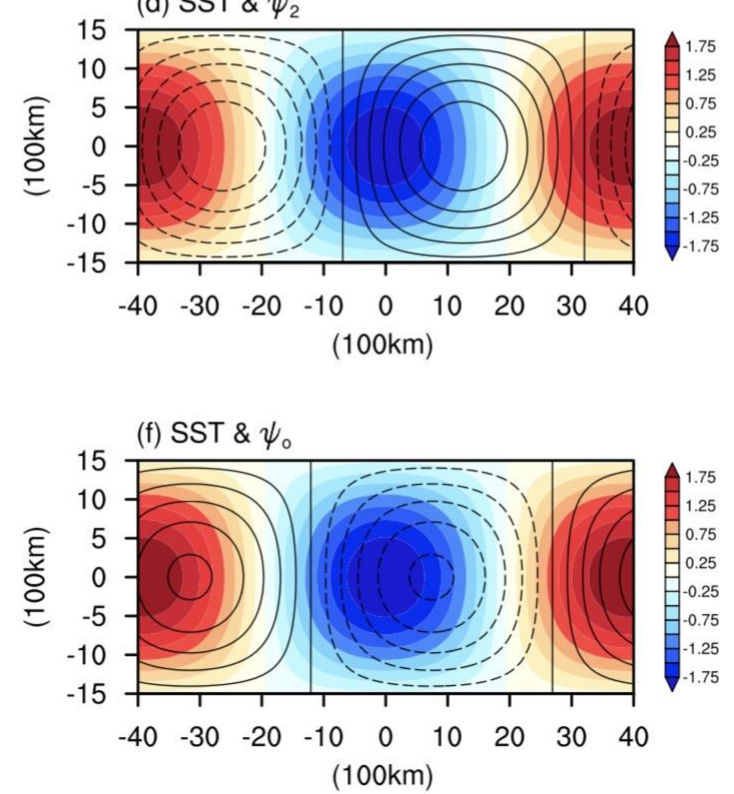

865 Figure 7. As in Fig. 4, but derived with (left) the surface diabatic heating alone and 866 (right) the mid-level diabatic heating alone. 
(a) upper Feddy

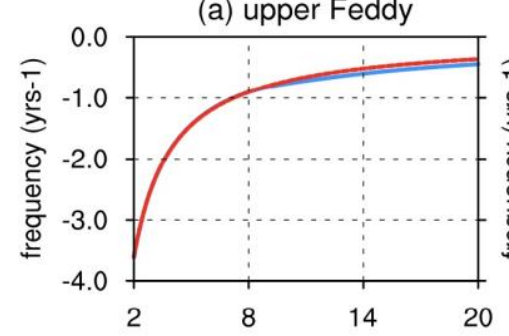

(d) upper Feddy

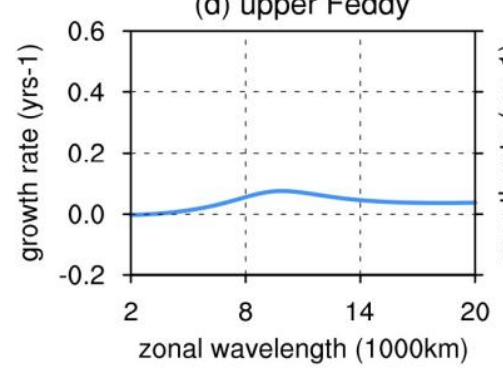

(b) lower Feddy

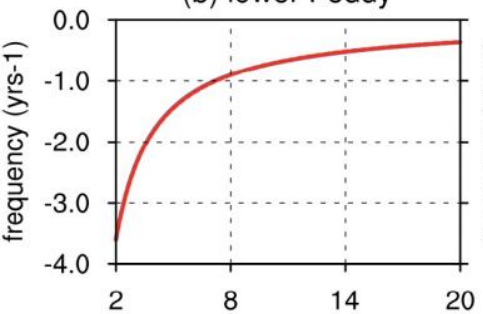

(e) lower Feddy

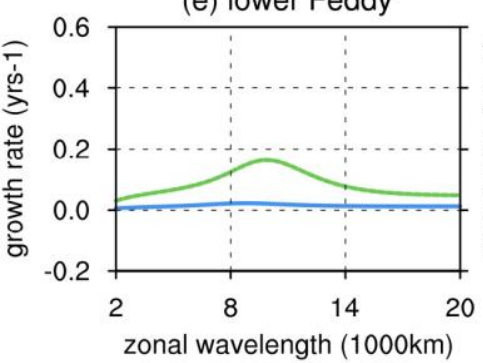

(c) upper+lower Feddy

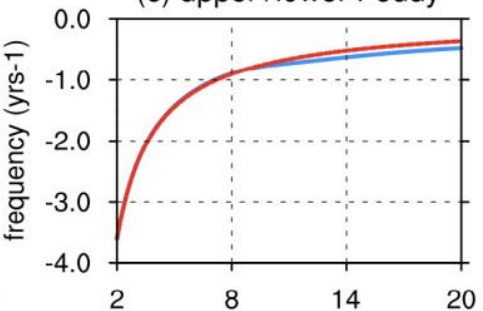

(f) upper+lower Feddy

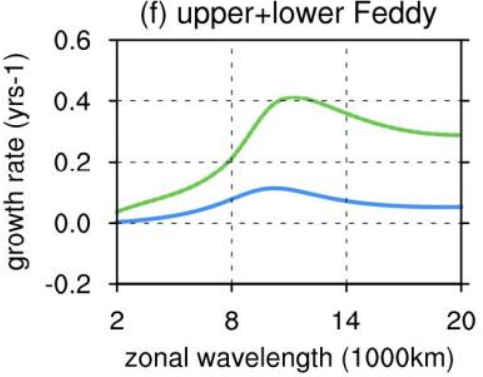

873 Figure 8. Frequency (a, b, c) and growth rate (blue lines in d, e, f) of the coupled

874 oceanic Rossby wave mode as a function of zonal wavelength corresponding to cases

875 when the transient eddy vorticity forcing occurs in lower layer only (a, d), in higher

876 layer only (b, e), and in both layers (c, f), respectively. Green lines in (e, f) indicate

877 the associated growth rate when the transient eddy vorticity forcing in lower layer is

878 increased to be equal to that in higher layer. Red lines in (a, c, e) denote the frequency

879 of free oceanic Rossby wave. 
upper Feddy

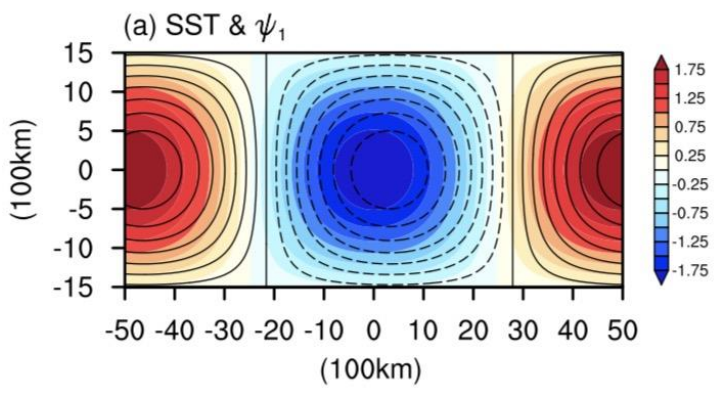

(c) SST \& $\psi_{2}$

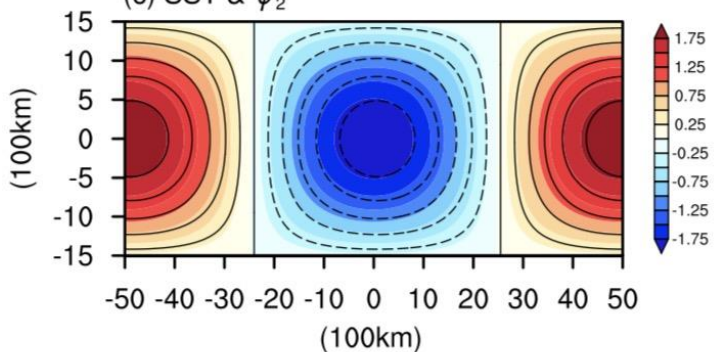

(e) SST \& $\psi_{0}$

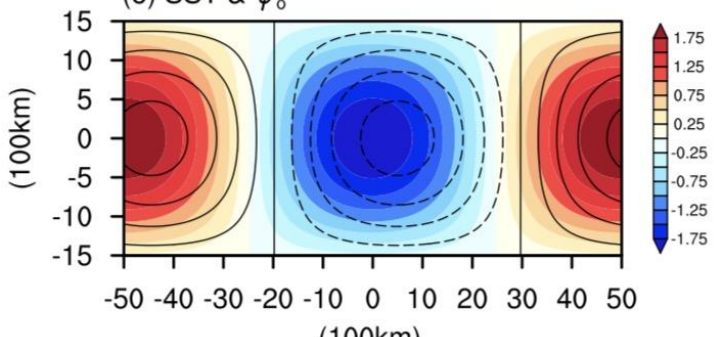

lower Feddy

(b) SST \& $\psi_{1}$

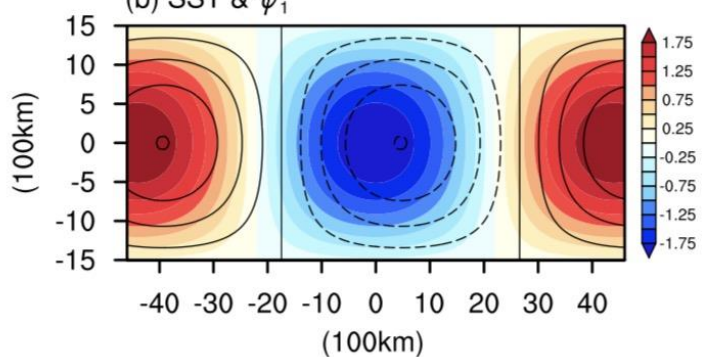

(d) SST \& $\psi_{2}$

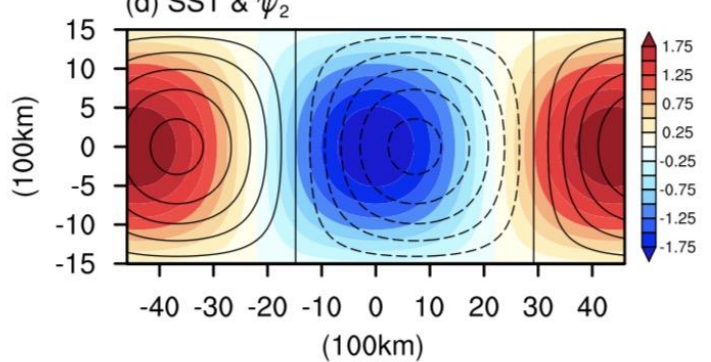

(f) SST \& $\psi$ 。

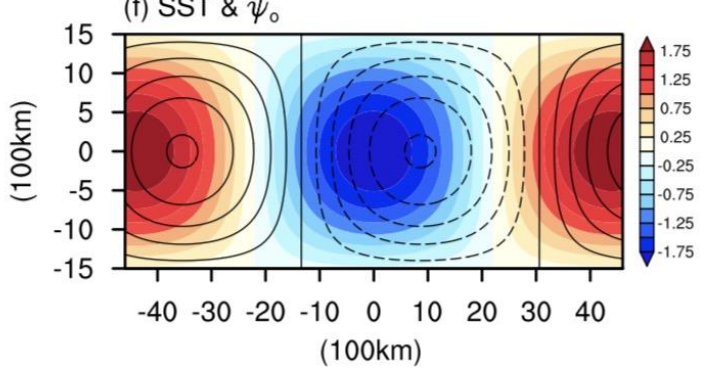

882 Figure 9. As in Fig. 4, but derived with (left) the lower-layer transient eddy vorticity

883 forcing alone and (right) the higher-layer transient eddy vorticity forcing alone. 
(a)

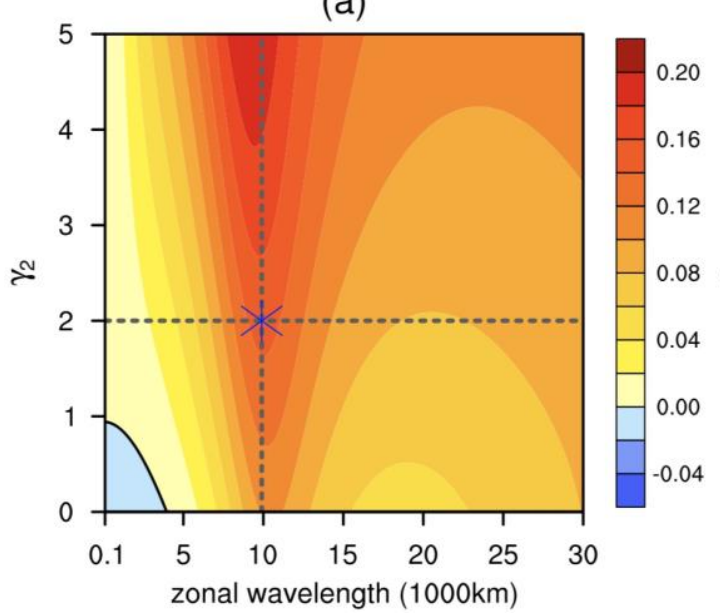

(b)

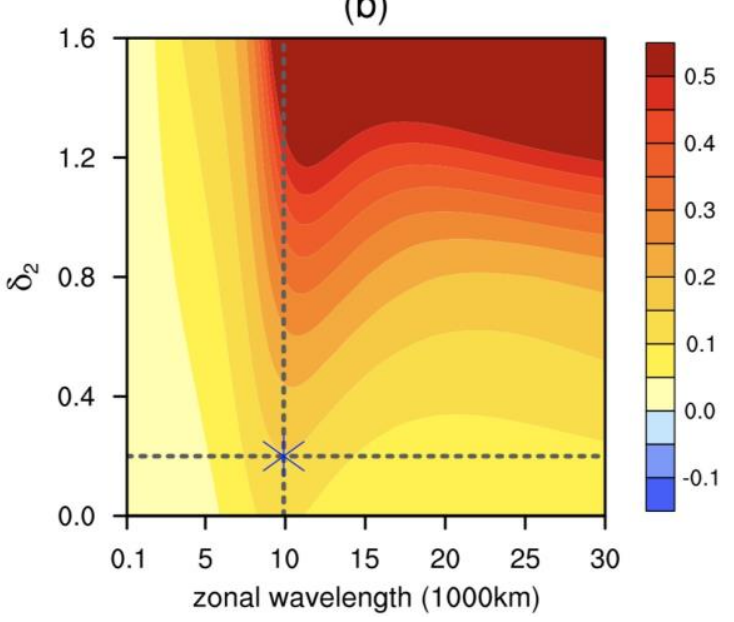

887 Figure 10. Unstable growth rate of coupled oceanic Rossby wave mode as a function

888 of zonal wavelength versus (a) the ratio of surface heating to mid-level heating $\left(\gamma_{2}\right.$,

889 here the mid-level heating is fixed and the surface heating is enhanced with the

890 increase of $\gamma_{2}$ ), and (b) the ratio of lower-layer transient eddy vorticity forcing to

891 higher-layer vorticity forcing $\left(\delta_{2}\right.$, here the higher-layer vorticity forcing is fixed and

892 the lower-layer vorticity forcing is enhanced with the increase of $\delta_{2}$ ), respectively. 
894

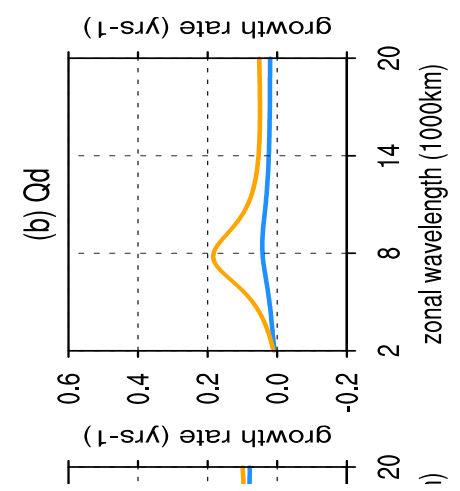

896 Figure 11. Comparison of the growth rate of the coupled oceanic Rossby wave mode

897 derived in our study with that derived in CYF2020 when (a) both diabatic heating and

898 transient eddy vorticity forcing are considered simultaneously, (b) the diabatic heating

899 is considered only and (c) the transient eddy vorticity forcing is considered only.

900 


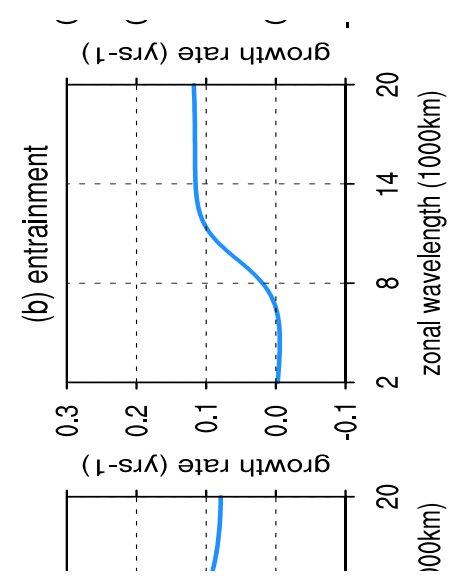

903 Figure 12. Growth rate as a function of wavelength for the coupled oceanic Rossby

904 wave mode with (a) full oceanic processes in SST evolution equation, (b)

905 entrainment- process only, and (c) advection process only. Note that (a) is the same as $906 \quad$ Fig. 3b.

907

908 
(a)

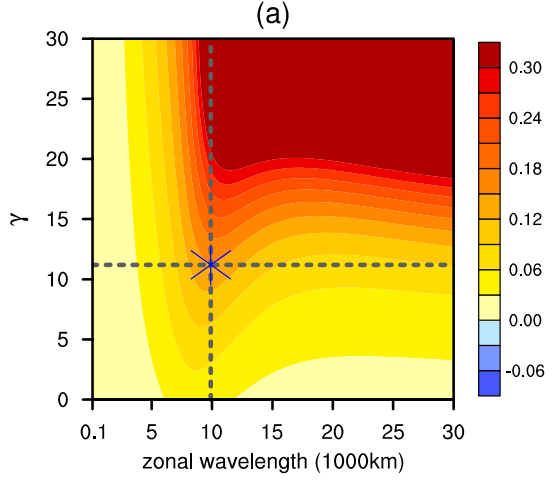

(c)

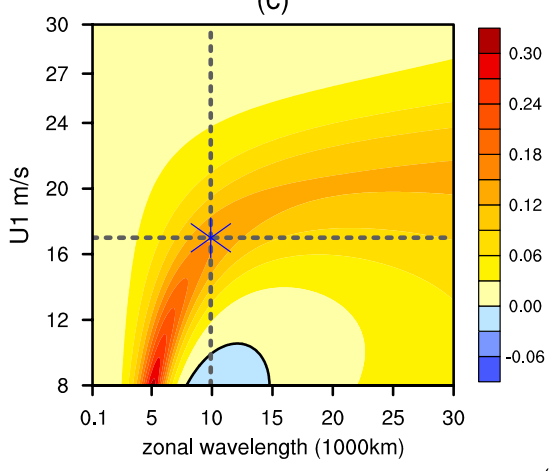

(b)

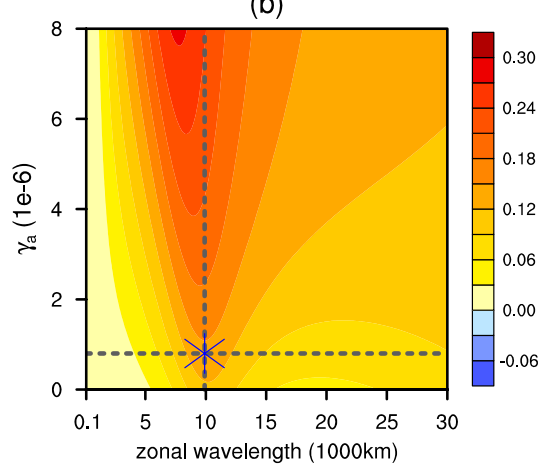

(d)

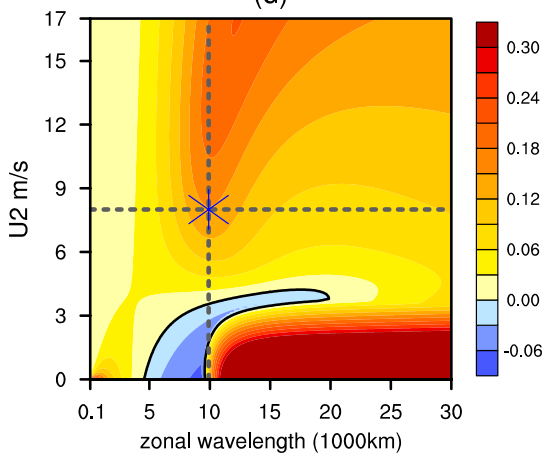

(e)

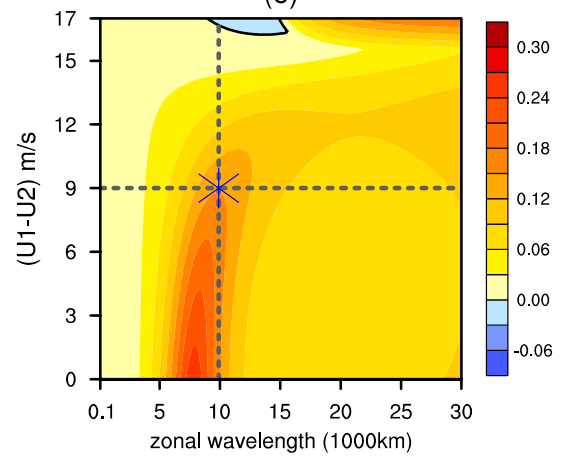

Figure 13. Unstable growth rate of coupled oceanic Rossby wave mode as a function

912 of zonal wavelength versus (a) dynamical coupling coefficient $(\gamma)$, (b) thermal

913 coupling coefficient $\left(\gamma_{a}\right)$, (c) the higher-layer background zonal wind $\left(U_{1}\right),(\mathrm{d})$ the

914 lower-layer background zonal wind $\left(U_{2}\right)$ and (e) the vertical wind shear $\left(U_{1}-U_{2}\right)$,

915 respectively.

916 


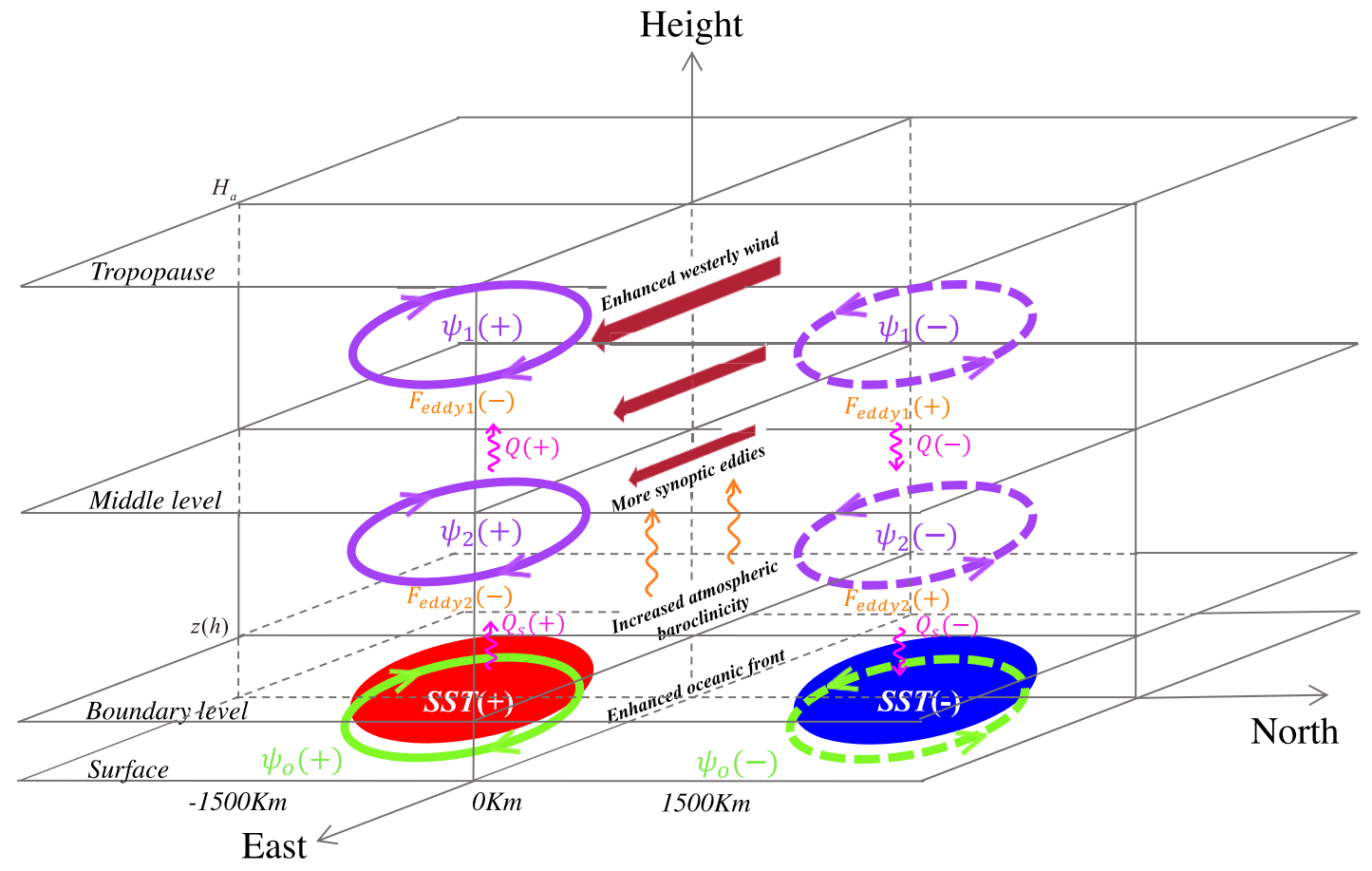

917

918 Figure 14. Schematic diagram of the phase relationships among atmospheric and

919 oceanic variables for the most unstable coupled oceanic Rossby wave mode in the 920 midlatitudes.

921

922 
Figures

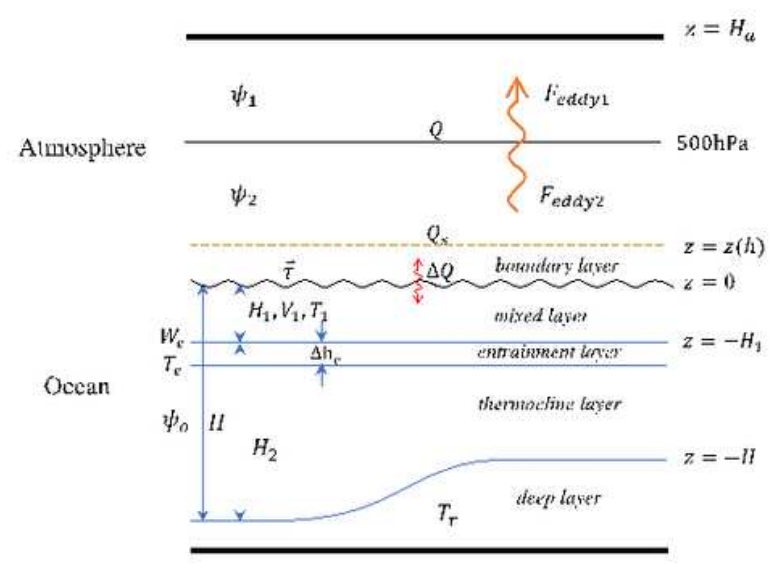

Figure 1

See the Supplemental Files section for the complete figure caption 
(a) geopotential height \& SST

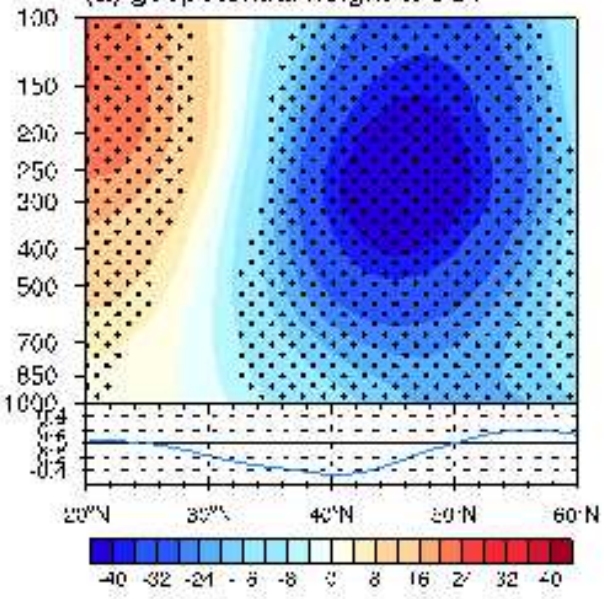

(b) $O d$

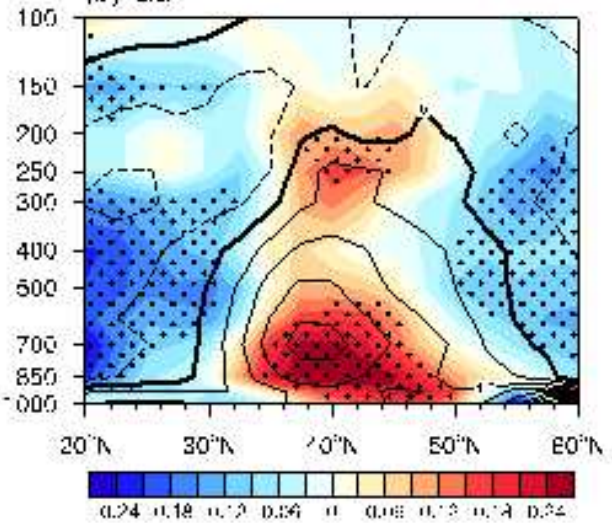

(c) Feddy

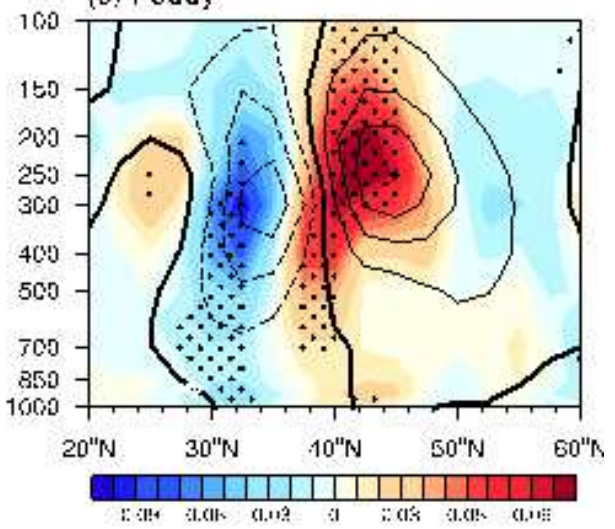

Figure 2

See the Supplemental Files section for the complete figure caption 
(a)

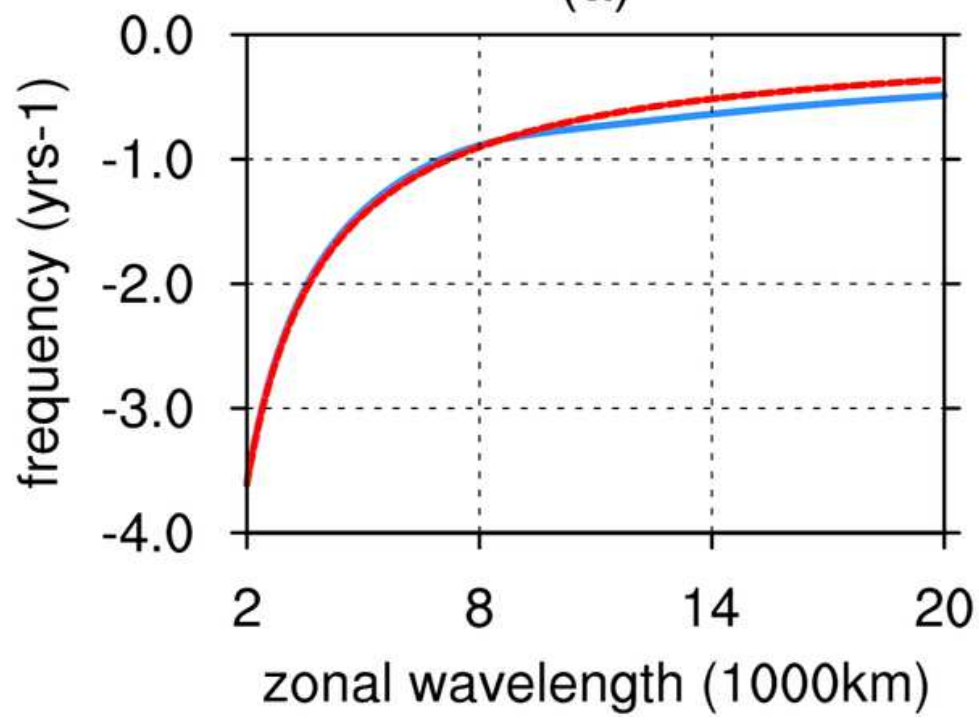

(b)

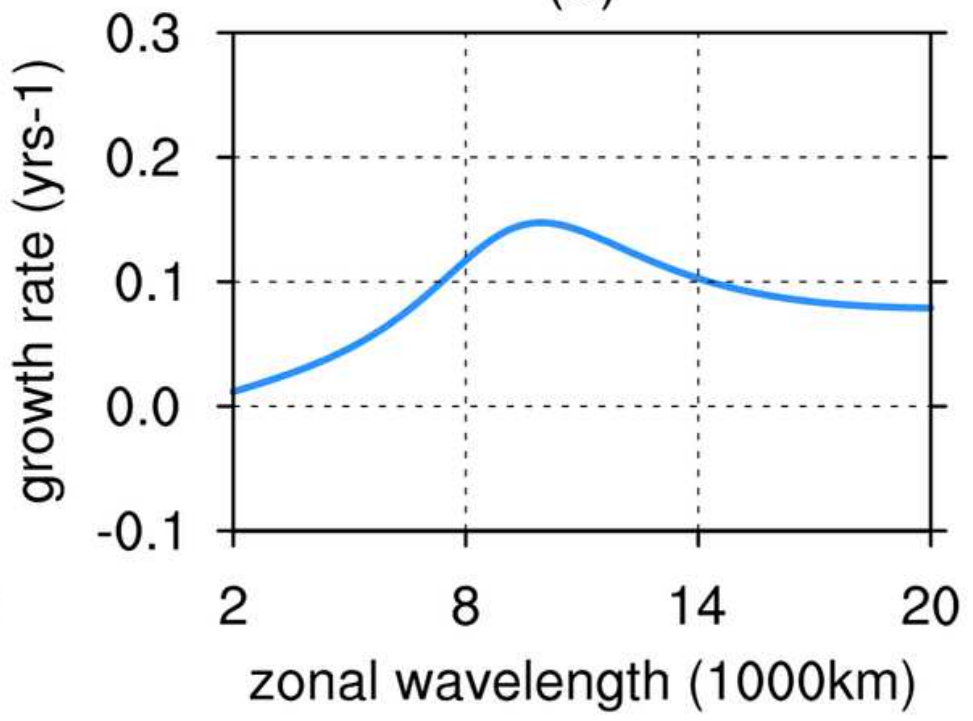

Figure 3

See the Supplemental Files section for the complete figure caption 

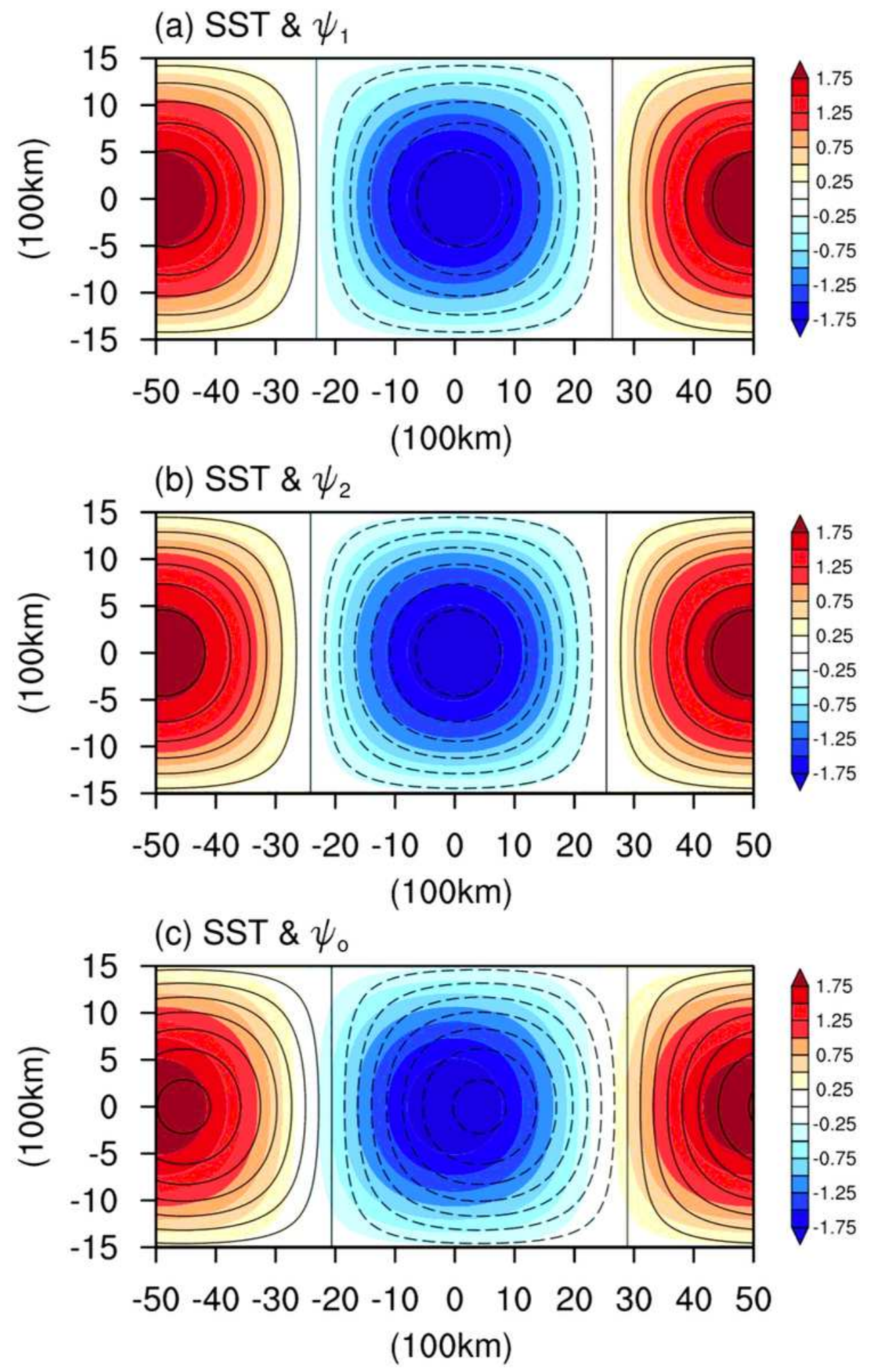

Figure 4

See the Supplemental Files section for the complete figure caption 
(a) SST \& $\psi_{\mathrm{a}}$

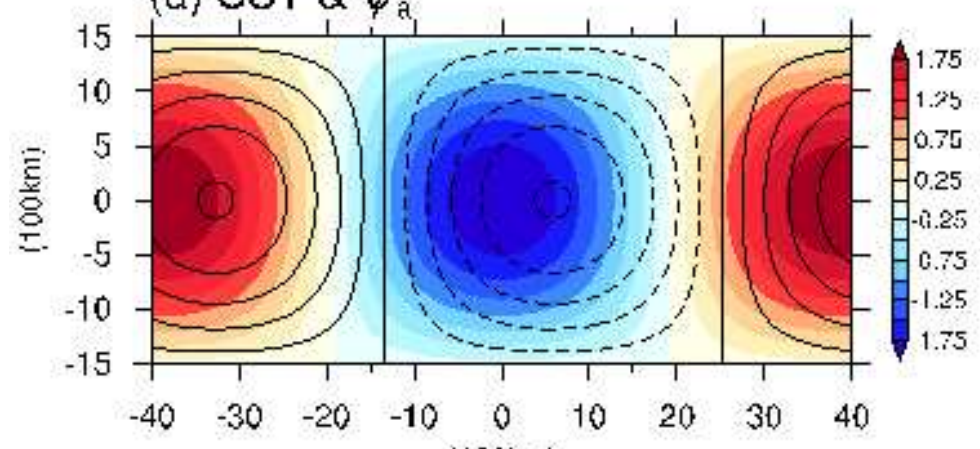

(c) SST \& $\psi_{\text {a }}$ regressed by PDO

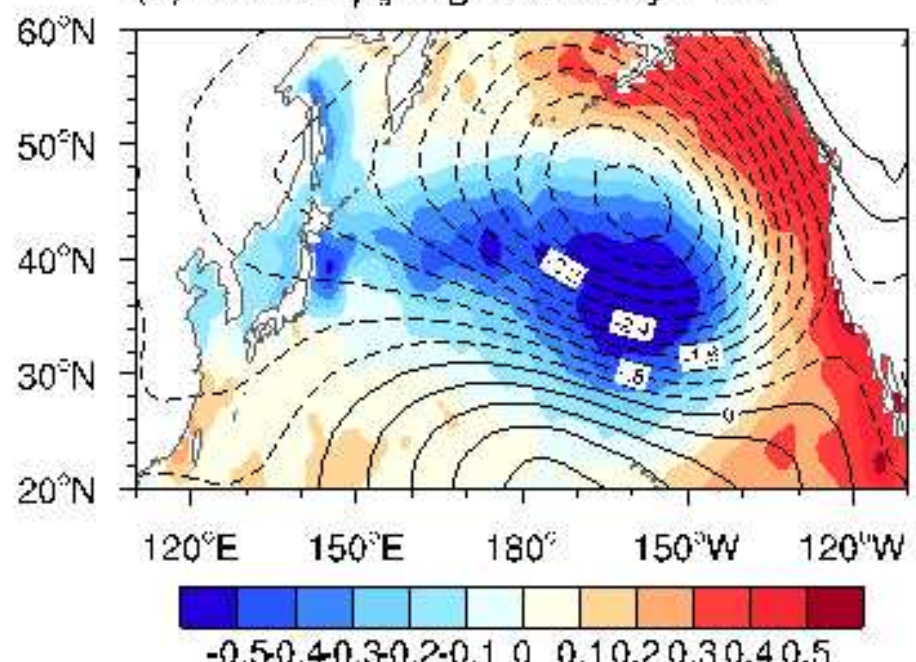

$-0.5-0.40 .30 .2-0.100 .10 .20 .30 .40 .5$ (b) SST \& $\%$

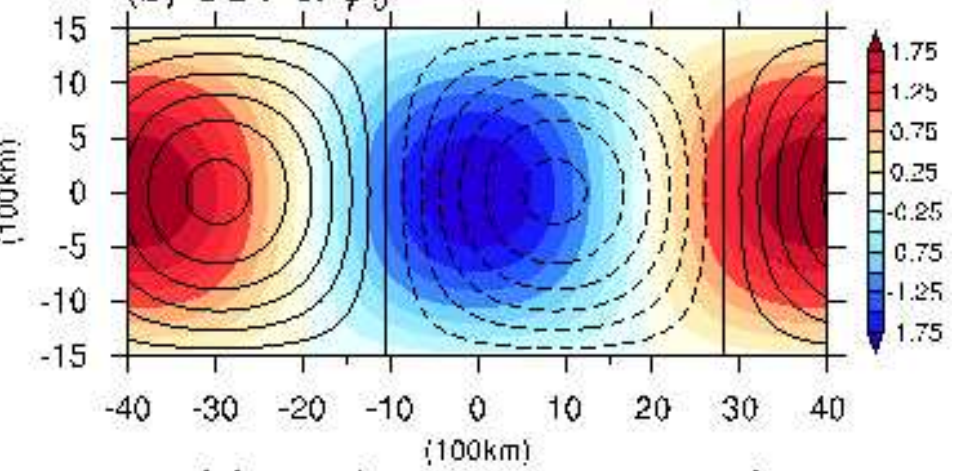

(d) SST \& $\psi_{0}$ regressed by PDO

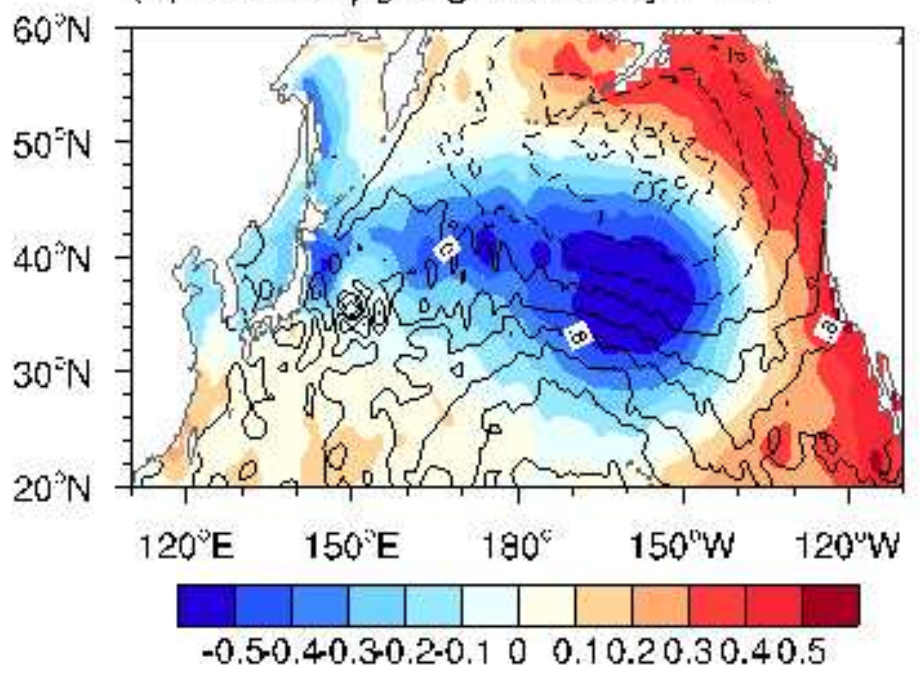

\section{Figure 5}

See the Supplemental Files section for the complete figure caption 
(a) Qs

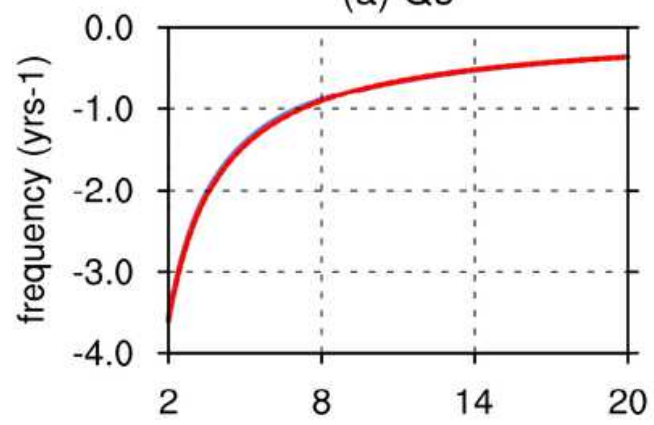

(b) Qs

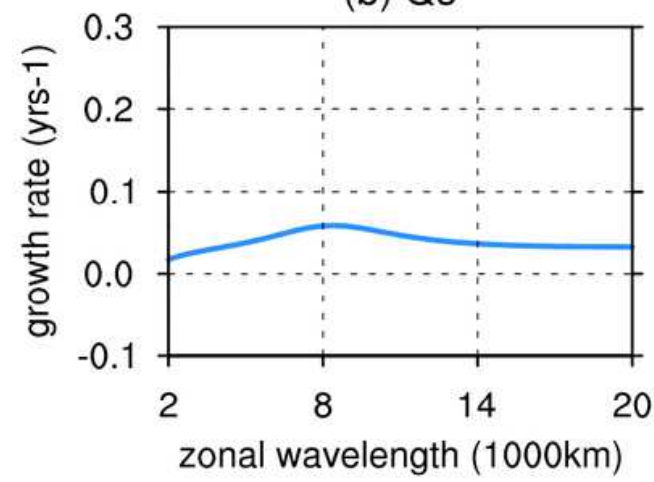

Figure 6

See the Supplemental Files section for the complete figure caption (e) Q+Qs
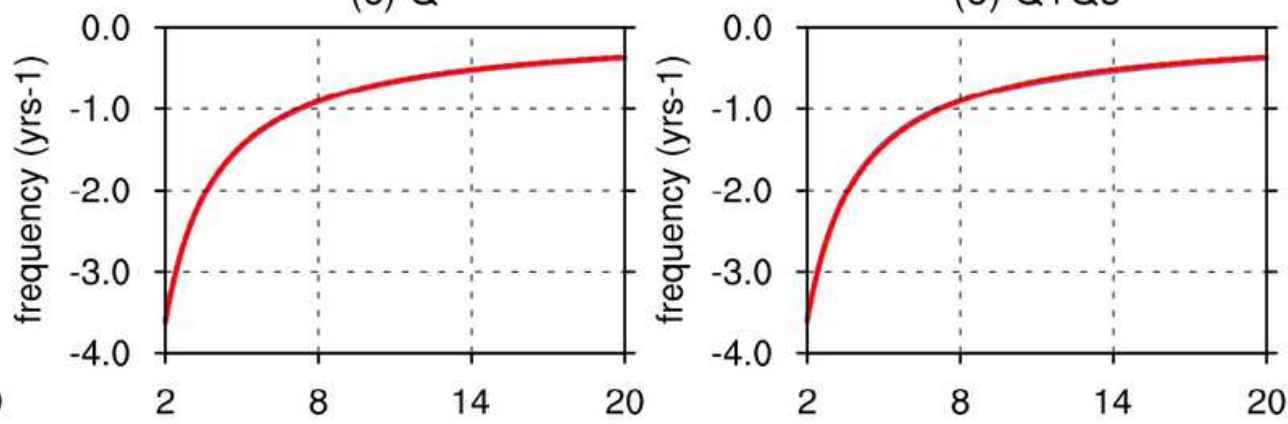

(d) $Q$
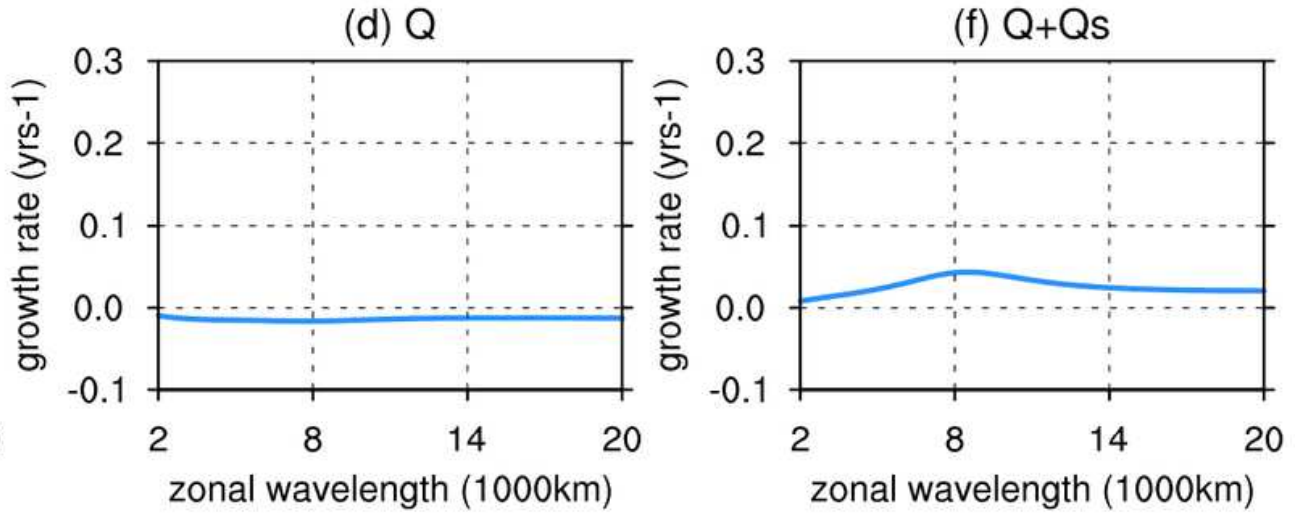
Qs

(a) SST \& $\psi_{1}$

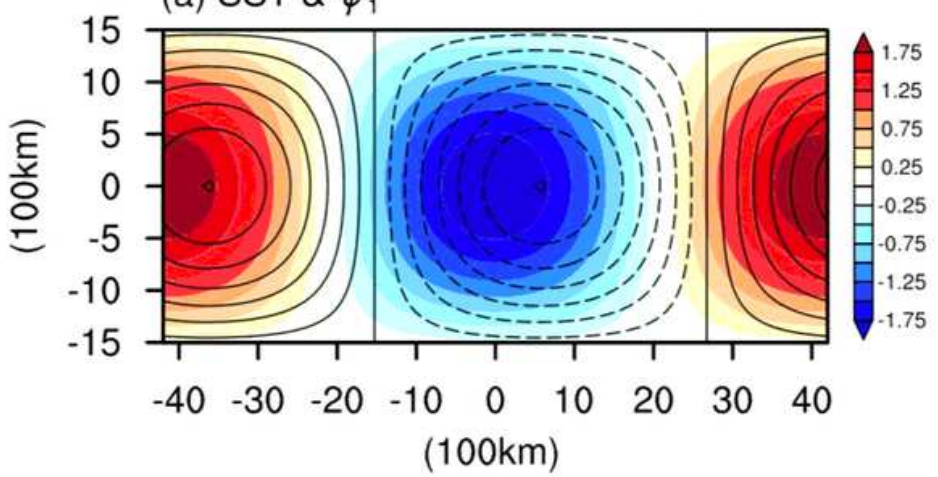

(c) SST \& $\psi_{2}$

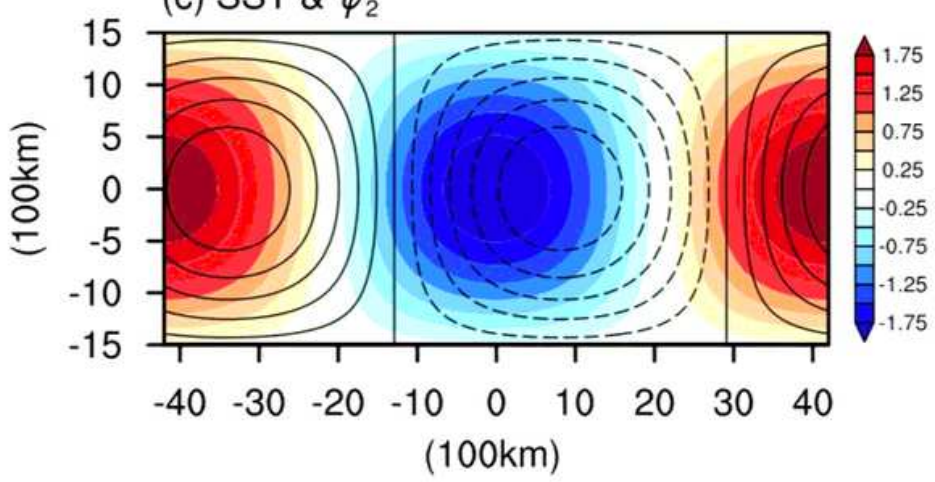

(e) SST \& $\psi_{\circ}$

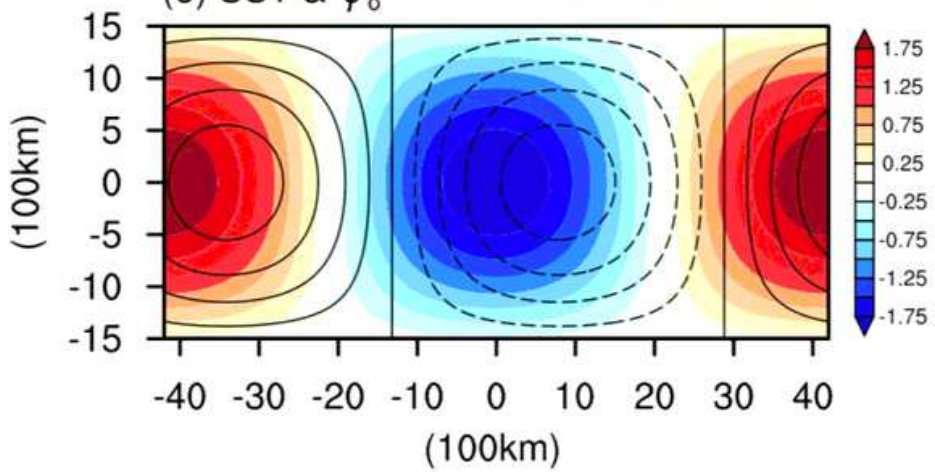

(b) SST \& $\psi_{1}$

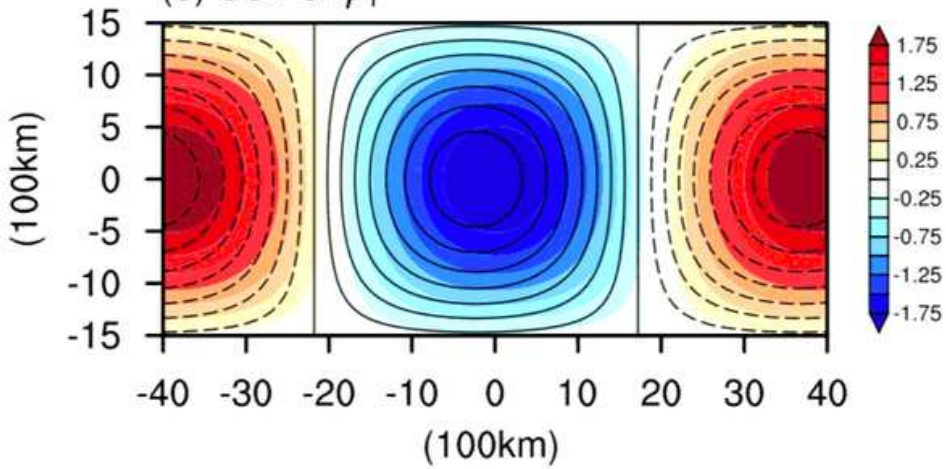

(d) SST \& $\psi_{2}$

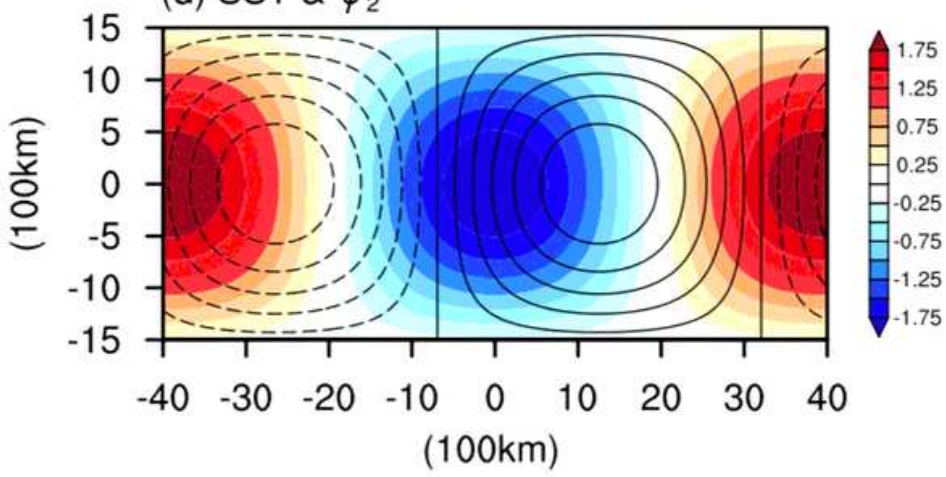

(f) SST \& $\psi$ 。

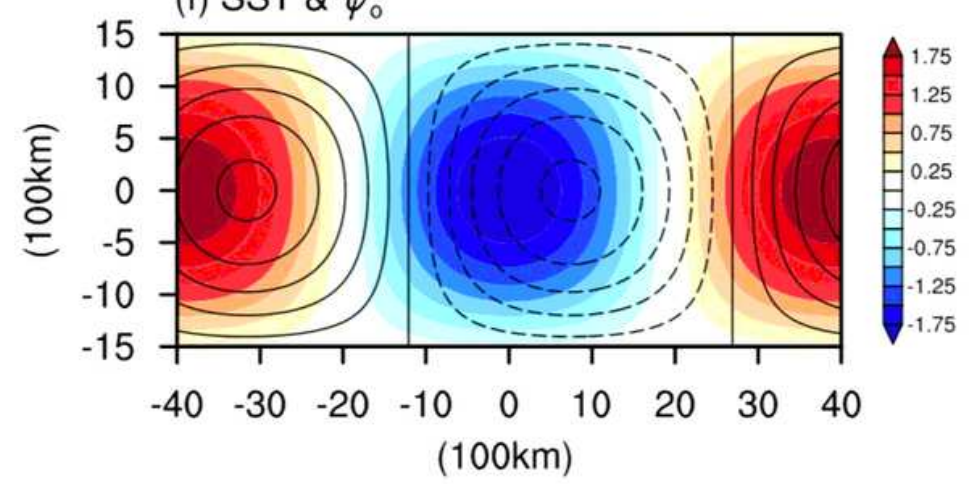

Figure 7

See the Supplemental Files section for the complete figure caption 


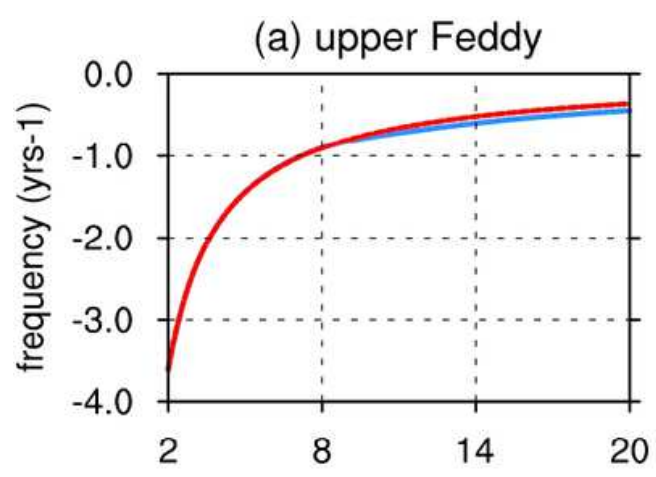

(d) upper Feddy

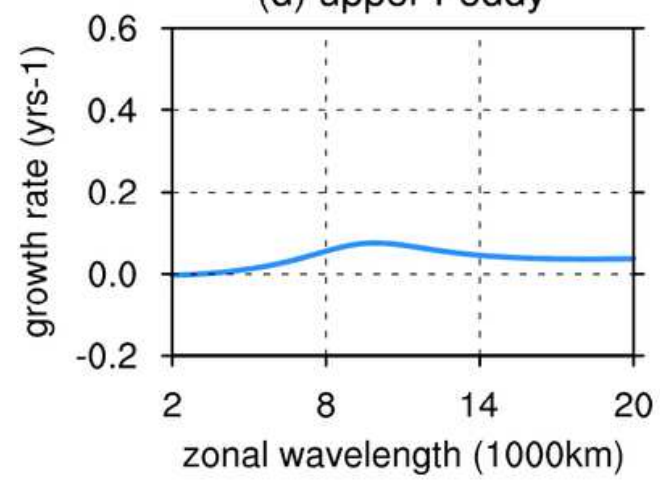

See the Supplemental Files section for the complete figure caption

\section{Figure 8}

(c) upper+lower Feddy
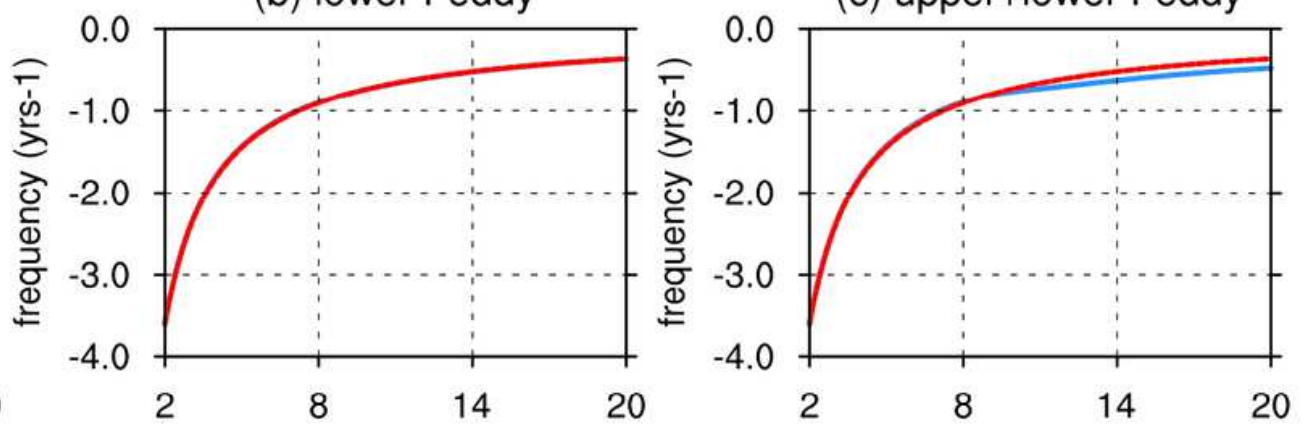

(e) lower Feddy
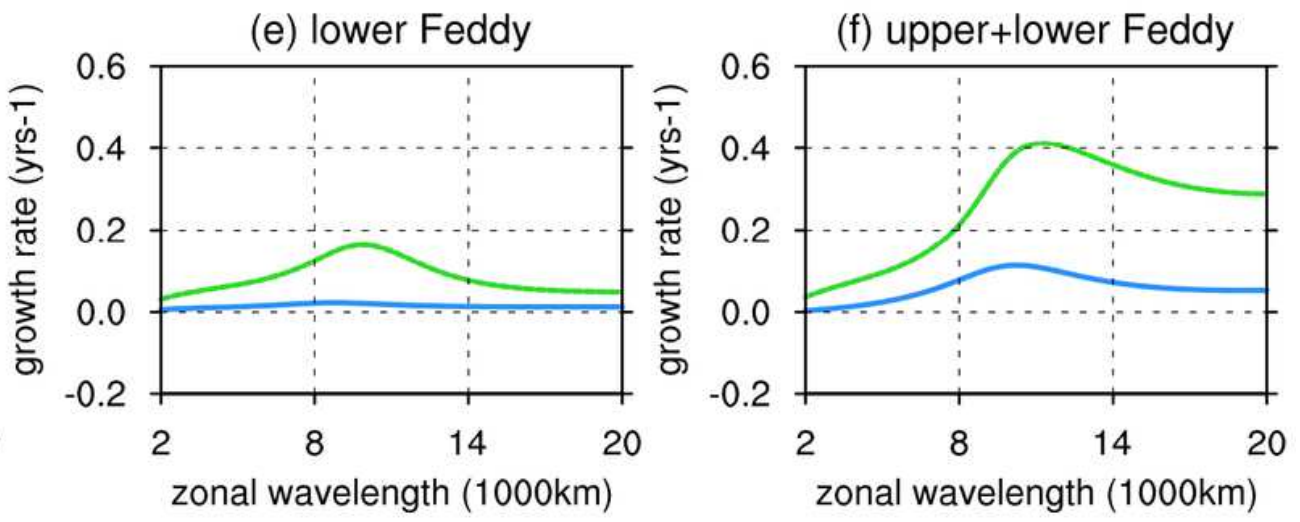
upper Feddy

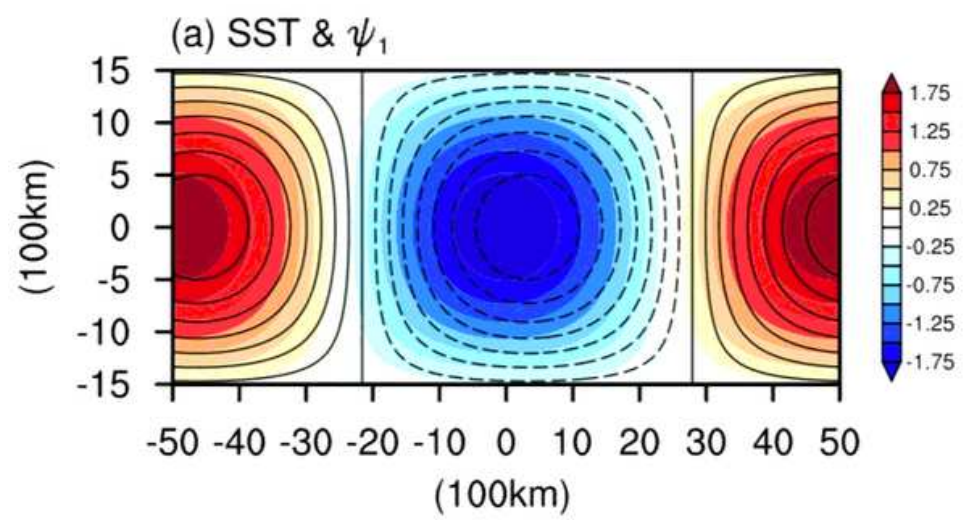

(c) SST \& $\psi_{2}$

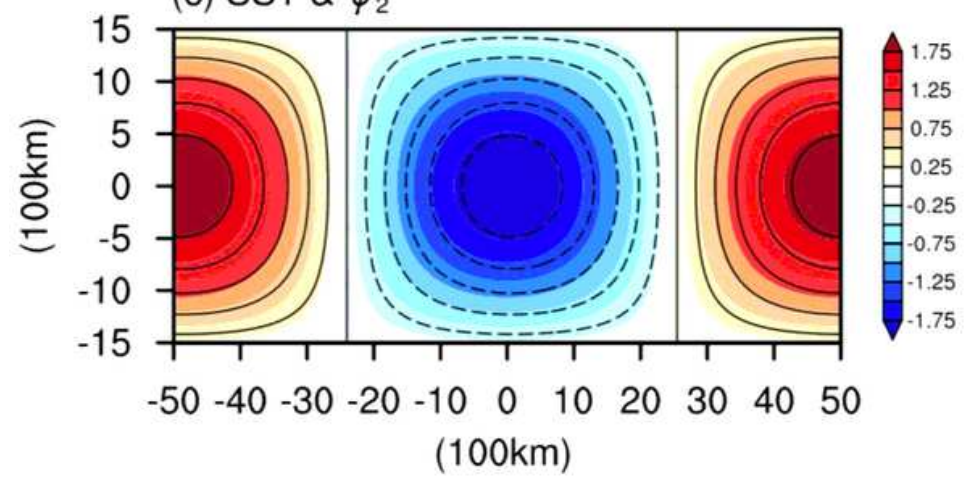

(e) SST \& $\psi_{\circ}$

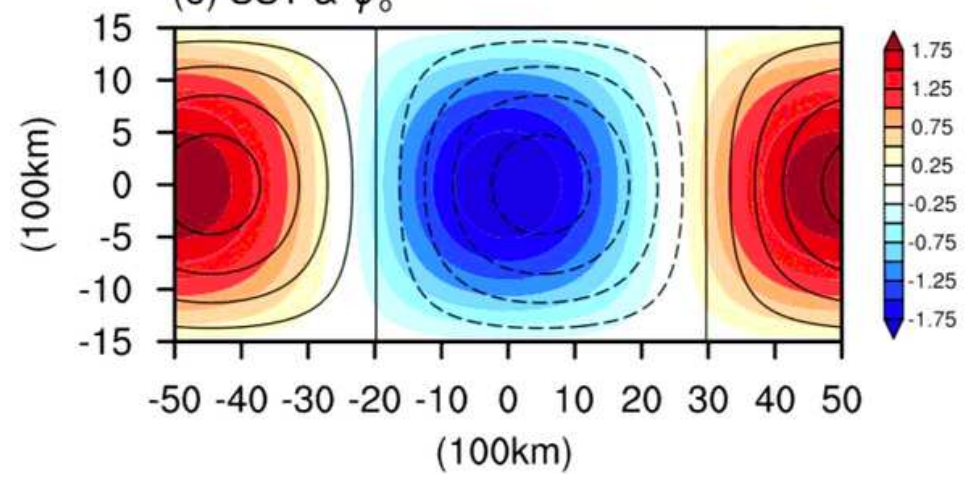

lower Feddy

(b) SST \& $\psi_{1}$

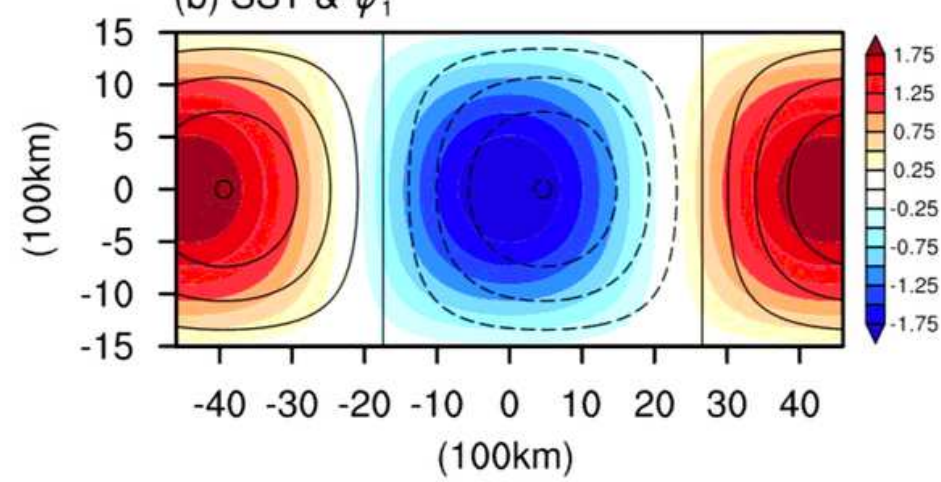

(d) SST \& $\psi_{2}$

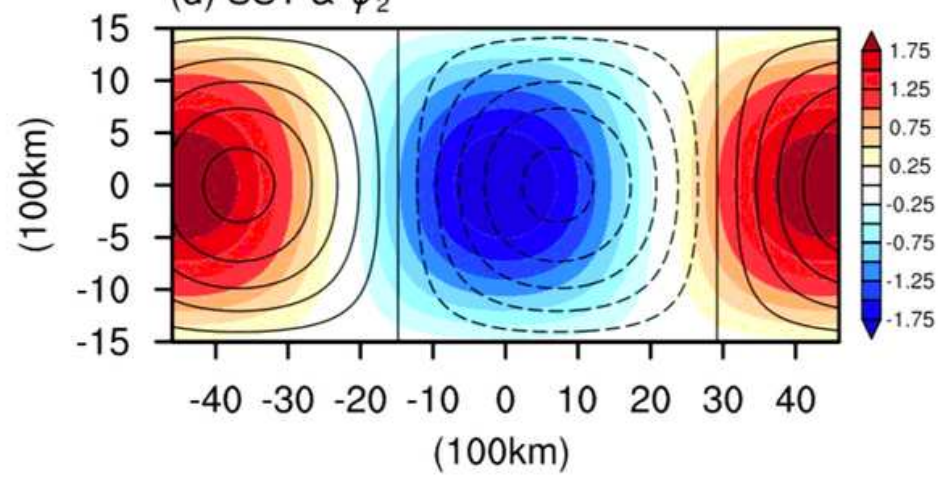

(f) SST \& $\psi$ 。

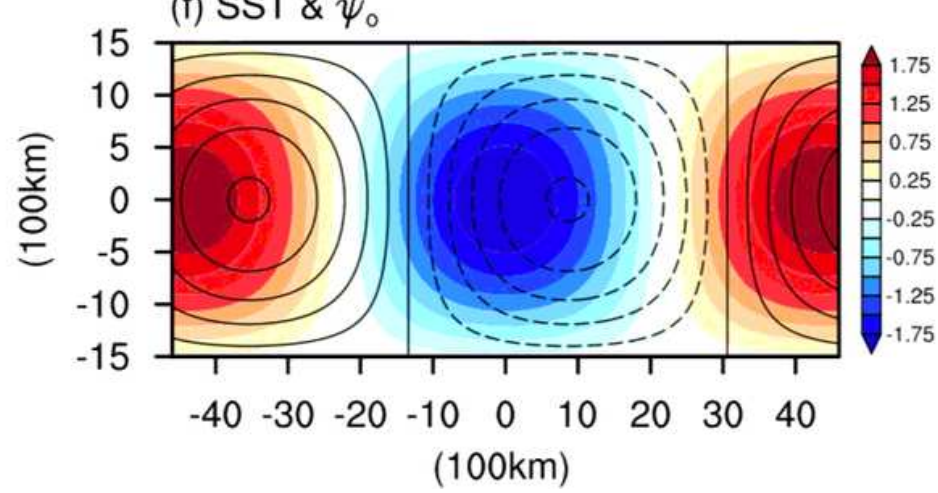

Figure 9

See the Supplemental Files section for the complete figure caption 
(a)

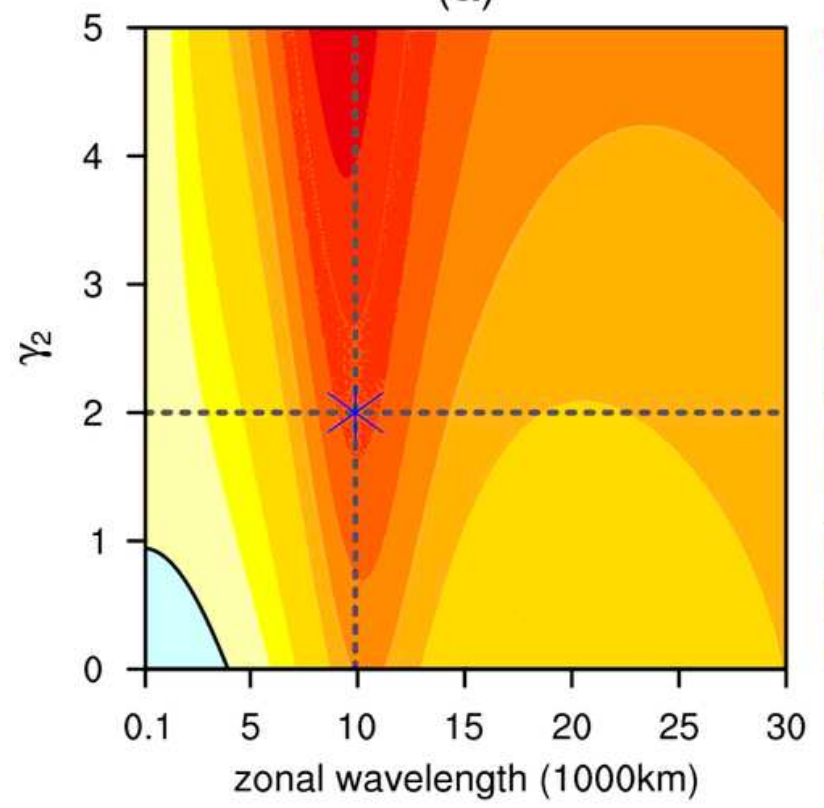

(b)

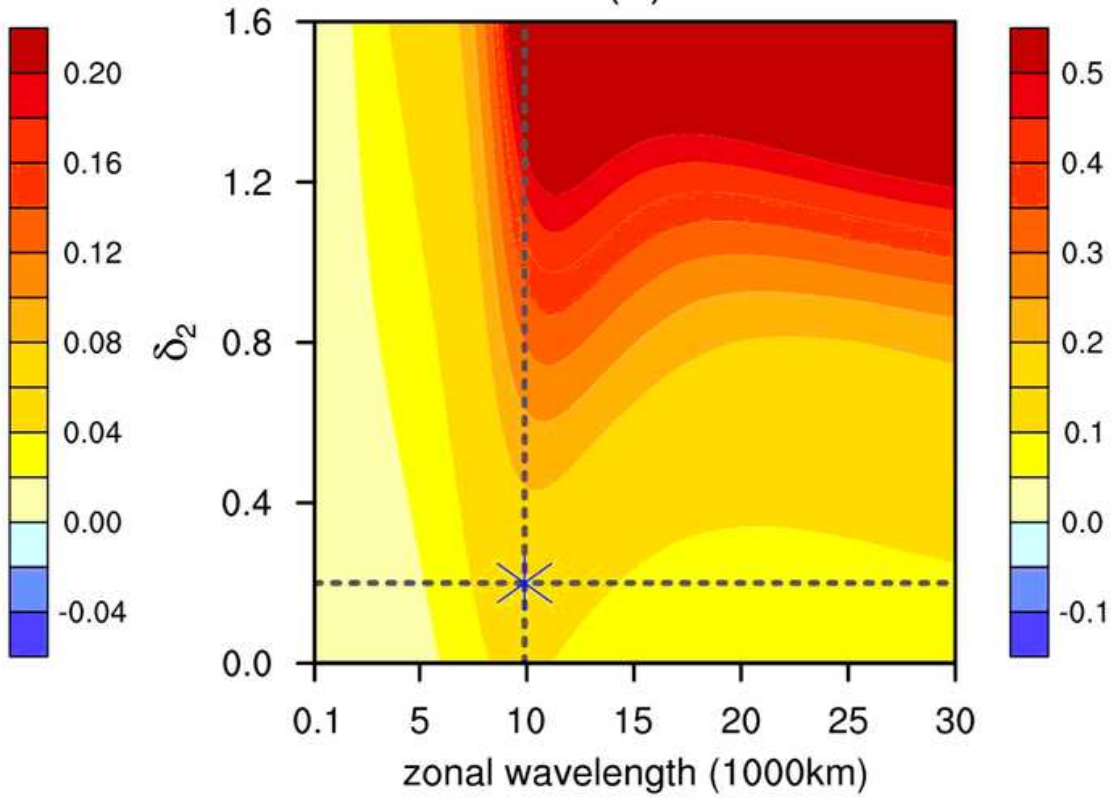

Figure 10

See the Supplemental Files section for the complete figure caption 

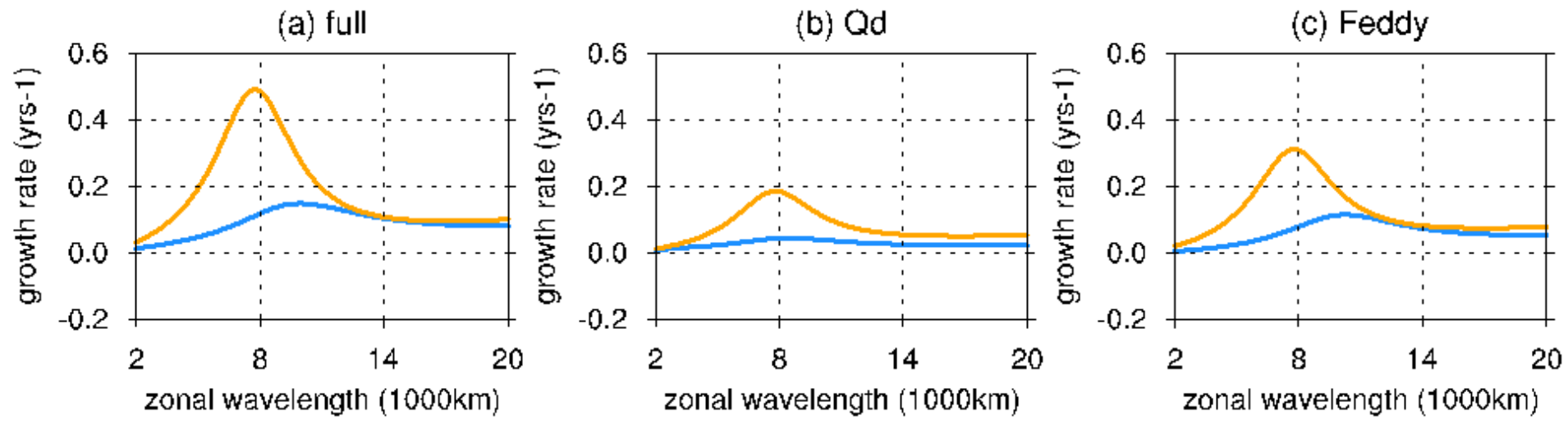

Figure 11

See the Supplemental Files section for the complete figure caption 

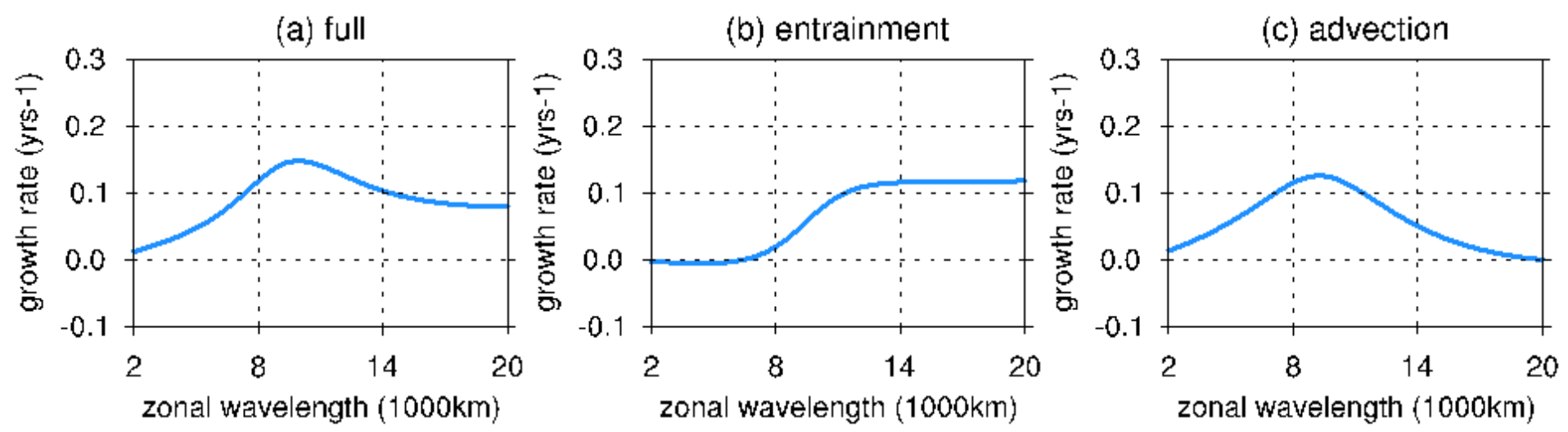

\section{Figure 12}

See the Supplemental Files section for the complete figure caption 
(a)

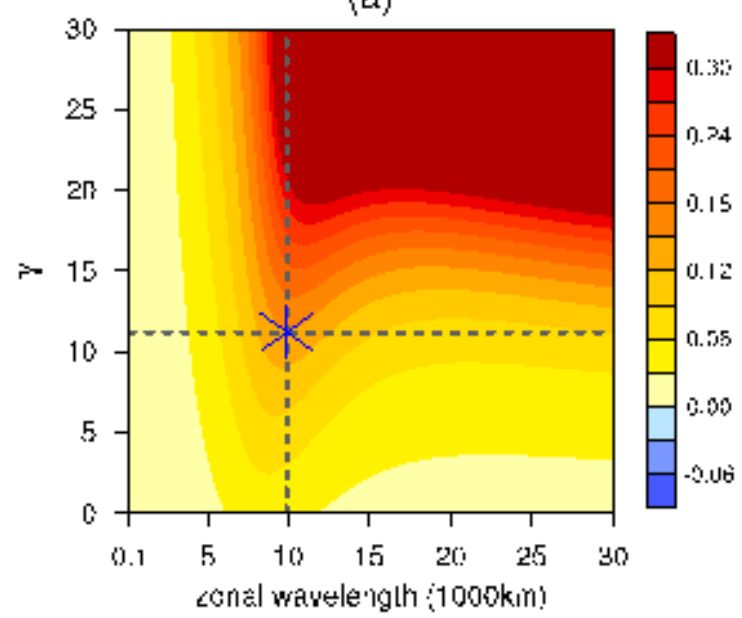

(c)

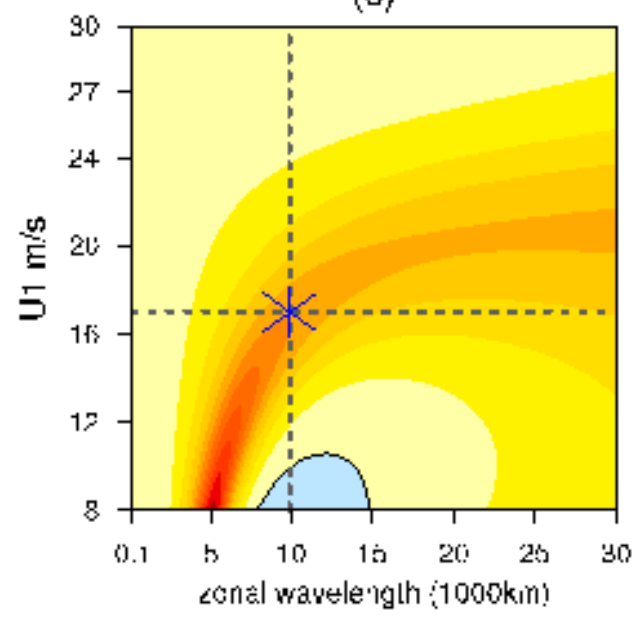

(b)

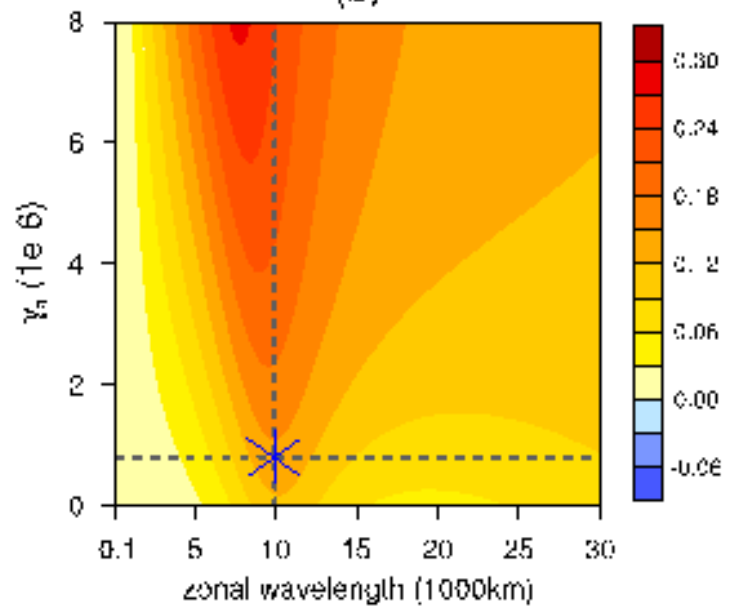

(d)

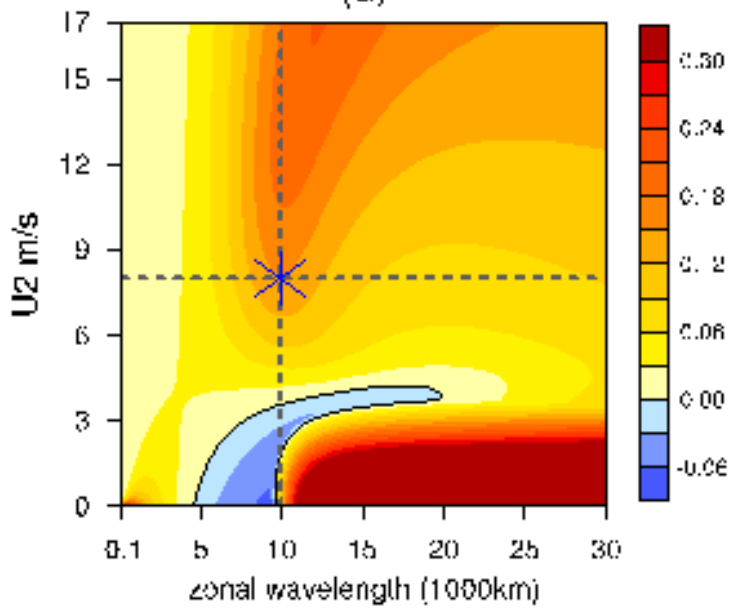

(e)

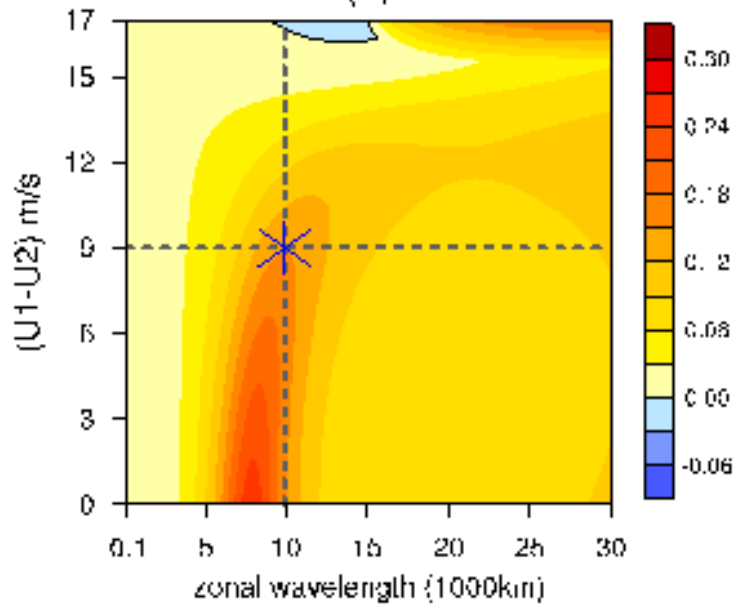

Figure 13

See the Supplemental Files section for the complete figure caption 


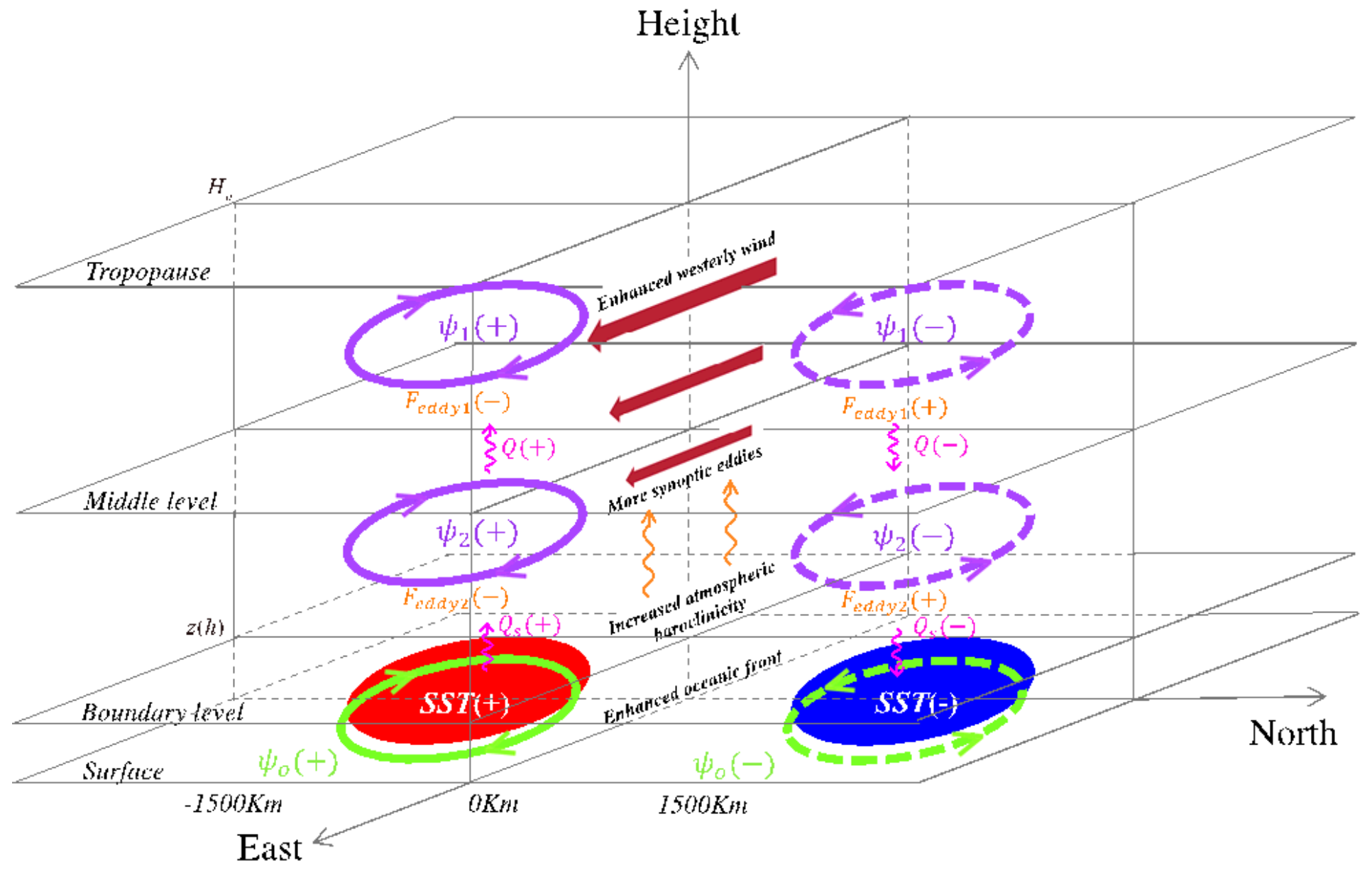

Figure 14

See the Supplemental Files section for the complete figure caption

\section{Supplementary Files}

This is a list of supplementary files associated with this preprint. Click to download.

- FigureCaptions.docx 